\title{
Globular clusters in the inner Galaxy classified from dynamical orbital criteria
}

\author{
Angeles Pérez-Villegas ${ }^{\oplus},{ }^{1 \star}$ Beatriz Barbuy ${ }^{\circledR},{ }^{1 \star}$ Leandro O. Kerber ${ }^{\circledR},{ }^{2}$ \\ Sergio Ortolani ${ }^{\circledR},{ }^{3}$ Stefano O. Souza ${ }^{\oplus 1}$ and Eduardo Bica ${ }^{\oplus 4}$ \\ ${ }^{1}$ Universidade de São Paulo, IAG, Rua do Matão 1226, Cidade Universitária, São Paulo 05508-900, Brazil \\ ${ }^{2}$ Universidade Estadual de Santa Cruz, Departamento de Ciências Exatas e Tecnológicas, Rodovia Jorge Amado km 16, Ilhéus 45662-000, Brazil \\ ${ }^{3}$ Dipartimento di Fisica e Astronomia 'Galileo Galilei', Università di Padova, Vicolo dell'Osservatorio 3, Padova I-35122, Italy \\ ${ }^{4}$ Departamento de Astronomia, Universidade Federal do Rio Grande do Sul, CP 15051, Porto Alegre 91501-970, Brazil
}

Accepted 2019 November 8. Received 2019 November 4; in original form 2019 August 2

\begin{abstract}
Globular clusters (GCs) are the most ancient stellar systems in the Milky Way. Therefore, they play a key role in the understanding of the early chemical and dynamical evolution of our Galaxy. Around 40 per cent of them are placed within $\sim 4 \mathrm{kpc}$ from the Galactic centre. In that region, all Galactic components overlap, making their disentanglement a challenging task. With Gaia Data Release 2, we have accurate absolute proper motions for the entire sample of known GCs that have been associated with the bulge/bar region. Combining them with distances, from RR Lyrae when available, as well as radial velocities from spectroscopy, we can perform an orbital analysis of the sample, employing a steady Galactic potential with a bar. We applied a clustering algorithm to the orbital parameters apogalactic distance and the maximum vertical excursion from the plane, in order to identify the clusters that have high probability to belong to the bulge/bar, thick disc, inner halo, or outer halo component. We found that $\sim 30$ per cent of the clusters classified as bulge GCs based on their location are just passing by the inner Galaxy, they appear to belong to the inner halo or thick disc component, instead. Most GCs that are confirmed to be bulge GCs are not following the bar structure and are older than the epoch of the bar formation.
\end{abstract}

Key words: Galaxy: bulge-globular clusters: general-Galaxy: kinematics and dynamics.

\section{INTRODUCTION}

The orbital evolution of globular clusters (GCs) in the Galaxy, combined with kinematics and stellar population analyses, can provide important information to decipher the history of our Galaxy. In Bica, Ortolani \& Barbuy (2016), 43 clusters were selected as related to the bulge component based on their location. Recently, Bica et al. (2019) compiled 200 GCs in the Galaxy, adding new entries such as the recently detected ones in the Vista Variables in the Via Lactea (Saito et al. 2012) survey by Minniti, AlonsoGarcía \& Pullen (2017a) and Minniti et al. (2017b). By including confirmed GCs plus candidates, the number rises to 294 objects, thus reducing the lack of the Galactic GCs with respect to M31 (Caldwell \& Romanowsky 2016).

The stellar population components in the Galaxy have been under scrutiny in the last decades. The Galactic disc and halo are well established, and the existence of a thick disc is increasingly more confirmed. As for the Galactic bulge configuration as an entity, it still

^E-mail: mperez@iag.usp.br (AP-V); b.barbuy@iag.usp.br (BB) is under definition. In early work, a sample of metal-rich GCs with a flattened distribution were identified as disc GCs by Zinn (1980, 1985). The properties of this disc system of metal-rich GCs were described by Armandroff (1989), to have a scale height between 0.8 and $1.5 \mathrm{kpc}$, a rotational velocity of $193 \pm 29 \mathrm{~km} \mathrm{~s}^{-1}$, and a line-of-sight velocity dispersion of $59 \pm 14 \mathrm{~km} \mathrm{~s}^{-1}$, concluding that these parameters are consistent with properties of thick-disc stars. Later, Frenk \& White (1982), Minniti (1995), and Côté (1999) deduced that metal-rich GCs within $3 \mathrm{kpc}$ from the Galactic centre, should be associated with the Galactic bulge rather than to the thick disc, based on kinematics, spatial distribution, and metallicity.

The Galactic bulge formation is under study in several contexts. Barbuy, Chiappini \& Gerhard (2018a) list the following possible scenarios: (a) bulge and thick disc were formed early on simultaneously through strong gas accretion, (b) hierarchical merging of subclumps, (c) merging of early thick-disc subclumps, (d) a major merger, (e) secular evolution of the bar, and (f) dwarf galaxies accretion. There is also the hypothesis of the oldest stellar populations in the Galactic bulge to be seen as an extension of the inner halo in the innermost Galaxy. 


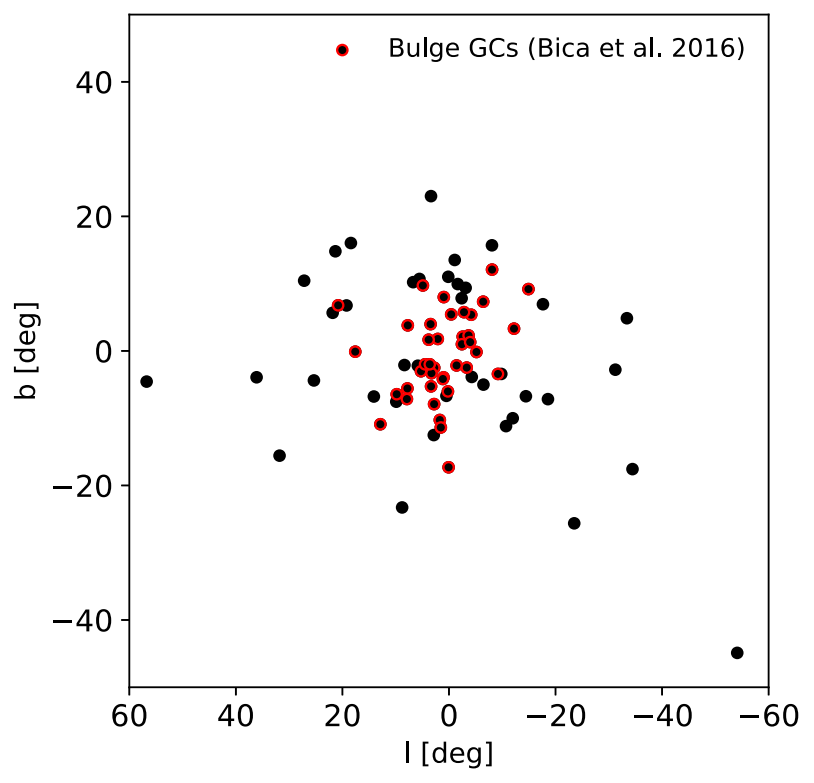

Figure 1. Location in Galactic coordinates of the GCs analysed. The red open circles are the clusters classified as bulge GCs in Bica et al. (2016).

The dynamical properties of GCs and their orbits provide further information that would help allocating the objects to a given stellar population. With the recently available Gaia Data Release 2 (DR2; Gaia Collaboration 2018a), the investigation of cluster orbits is made timely. Previously, this kind of work was attempted for the bulge clusters, by Dinescu et al. (2003). More recently, PérezVillegas et al. (2018) and Rossi et al. (2015b) derived proper motions and orbits for nine inner bulge clusters.

In this work, we study a sample of bulge GCs, plus the intruders or outsiders from Bica et al. (2016, their tables 1 and 2). In order to compute orbits, reliable radial velocities, proper motions, and distances are needed. Radial velocities, which can only be derived from spectroscopy, are still missing for about a dozen of these clusters, making that 78 of them can be studied. The dynamical parameters perigalactic and apogalactic distances, maximum height from the plane and eccentricity, are derived such that their appartenance to different Galaxy components are identified.

In Section 2, the sample and available data are described. In Section 3, the Galactic model potential is detailed. In Section 4, dynamical properties of the orbits are discussed, and in Section 5 the GCs are classified. The bulge GCs are further discussed in Section 6, and conclusions are drawn in Section 7.

\section{DATA}

The sample includes 40 GCs from the list of bulge GCs by Bica et al. (2016, their table 1), for which radial velocities are available. Another $37 \mathrm{GCs}$ from the outer shell, clusters somewhat farther, at distances $3.0<\mathrm{d}_{\mathrm{GC}}<4.5 \mathrm{kpc}$, some clusters with $\mathrm{d}_{\mathrm{GC}}>4.5 \mathrm{kpc}$ from the Galactic centre (Bica et al. 2016; their table 2), and the halo GC NGC 6752 are also added, with the purpose of identifying differences in the orbital properties among clusters that belong to each Galactic component. Fig. 1 shows the location in Galactic coordinates of our total sample that contains 78 clusters.

In Table 1, we list the cluster parameters employed in this study. Equatorial $(\alpha, \delta)$ and Galactic $(l, b)$ coordinates are given in Columns 2-5. The heliocentric distance $d_{\odot}$, in Column 6 is taken from colour-magnitude diagrams (CMDs) and RR Lyrae star distance determination for the clusters HP 1, Terzan 10, NGC 6522, NGC 6626, NGC 6558, Djorgovski 1, Djorgovski 2, NGC 6304, NGC 6624, NGC 6637, NGC 6652, NGC 6717, NGC 6723, and NGC 6362 (Kerber et al. 2018; Barbuy et al. 2018b; Kerber et al. 2019; Ortolani et al. 2019a,b; Oliveira et al., in preparation) that we consider reliable. For the others, distances from Harris (1996, edition of 2010, hereafter H10), or Baumgardt et al. (2019) were adopted. Radial velocities $V_{\mathrm{r}}$, given in Column 7 , are taken from spectroscopy analyses from the literature. The absolute proper motions (PMs), $\mu_{\alpha}^{*}=\mu_{\alpha} \cos \delta, \mu_{\delta}$, in Columns 8 and 9, are the values estimated from the Gaia DR2 (Gaia Collaboration 2018b) and for the clusters marked with a star we calculated the PMs following the procedure explained next, where the uncertainties of PMs include the systematic error of $0.035 \mathrm{mas} \mathrm{yr}^{-1}$ reported by the Gaia Collaboration (2018b). The metallicties, given in Column 10, available from high-resolution studies reported in Bica et al. (2016, their table 3), Barbuy et al. (2014, 2018b) for NGC 6522 and NGC 6558, derivations from literature and isochrone fittings to CMDs from our group (Oliveira et al., in preparation), and for the remaining clusters the values are adopted from the website by Bruno Dias. ${ }^{1}$ Column 11 gives the core radius taken from $\mathrm{H} 10$.

For the proper motion derivation from Gaia DR2, we selected the individual stars with the astrometric information including positions and PMs within 5 arcmin from the cluster centre. We removed the stars with $\mathrm{PM}$ errors $>0.25$ mas $\mathrm{yr}^{-1}$. Then, stars in each cluster were retrieved, within the core radius of each cluster from H10 to estimate the PMs. A selection of member stars was carried out with a combined plot of PMs in both directions, and through a Gaussian mixture model (GMM), as shown in Fig. 2, in order to get the mean PM in each direction and their uncertainties. We applied this method to $32 \mathrm{GCs}$ of our sample, and the PM determinations for them are inside $1 \sigma$ compared with those estimated by Gaia Collaboration (2018b), Vasiliev (2019), and Baumgardt et al. (2019), except for Terzan 1 and Terzan 5, that are inside $3 \sigma$. The PMs for the remaining clusters are taken from the new PM determination of GCs using Gaia DR2 from the references above.

In the past, the measurement of absolute PMs was a difficult task, particularly for GCs in the innermost Galaxy, and when available, the uncertainties were large, whereas now with Gaia that issue is essentially solved. Presently, the input distance values are the major source of uncertainty to construct more precise orbits along the Galaxy, and therefore in the classification of the GCs into a specific stellar population. The distance values for some of them, in particular for the most reddened ones, can be significantly different from H10 or Baumgardt et al. (2019) with their kinematic distance estimations.

Table 2 shows a compilation of distances from the literature. The distances used for the orbital integration are given in column 2, the distances from H10 catalogue are in column 3, the kinematic distances by Baumgardt et al. (2019) in column 4, distance determinations by Valenti, Ferraro \& Origlia (2007), Valenti, Ferraro \& Origlia (2010) in column 5, the distances estimated by the inverse of the parallax from Gaia Collaboration (2018b) are in column 6 , distances taken from isochrone fittings of the CMD and RR Lyrae estimated by our group are given in column

\footnotetext{
${ }^{1}$ http://www.sc.eso.org/ bdias/catalogues.html
} 
Table 1. Globular clusters data.

\begin{tabular}{|c|c|c|c|c|c|c|c|c|c|c|}
\hline Cluster & $\begin{array}{l}\alpha_{2000} \\
(\mathrm{deg})\end{array}$ & $\begin{array}{l}\delta_{2000} \\
(\mathrm{deg})\end{array}$ & $\begin{array}{c}1 \\
(\mathrm{deg})\end{array}$ & $\begin{array}{c}\mathrm{b} \\
(\mathrm{deg})\end{array}$ & $\begin{array}{c}\mathrm{d}_{\odot} \\
(\mathrm{kpc})\end{array}$ & $\begin{array}{c}V_{\mathrm{r}} \\
\left(\mathrm{km} \mathrm{s}^{-1}\right)\end{array}$ & $\begin{array}{c}\mu_{\alpha}^{*} \\
\left(\operatorname{mas~yr}^{-1}\right)\end{array}$ & $\begin{array}{c}\mu_{\delta} \\
\left(\operatorname{mas~yr}^{-1}\right)\end{array}$ & $\begin{array}{c}{[\mathrm{Fe} / \mathrm{H}]^{\dagger}} \\
(\mathrm{dex})\end{array}$ & $\begin{array}{c}\mathrm{R}_{c}^{\ddagger} \\
(\operatorname{arcmin})\end{array}$ \\
\hline BH 261 & 273.52 & -28.63 & 3.36 & -5.27 & $6.50 \pm 0.65^{e}$ & $-29.38 \pm 0.60^{e}$ & $3.59 \pm 0.05$ & $-3.57 \pm 0.05$ & -1.17 & 0.40 \\
\hline Djorg 1 & 266.86 & -33.06 & 356.67 & -2.48 & $9.30 \pm 0.50^{a}$ & $-358.10 \pm 0.70^{i}$ & $-5.11 \pm 0.07$ & $-8.30 \pm 0.05$ & -1.36 & 0.50 \\
\hline ESO452SC11 & 249.85 & -28.39 & 351.91 & 12.09 & $6.50 \pm 0.65^{e}$ & $16.27 \pm 0.48^{e}$ & $-1.54 \pm 0.05$ & $-6.41 \pm 0.05$ & -1.47 & 0.50 \\
\hline HP $1^{\star}$ & 262.77 & -29.98 & 357.42 & 2.12 & $6.59 \pm 0.16^{c}$ & $40.00 \pm 0.50^{j}$ & $2.41 \pm 0.06$ & $-10.14 \pm 0.05$ & $-1.00^{m}$ & 0.03 \\
\hline Liller 1 & 263.35 & -33.38 & 354.84 & -0.16 & $8.20 \pm 0.82^{d}$ & $60.18 \pm 2.46^{e}$ & $-5.53 \pm 0.52$ & $-7.69 \pm 0.33$ & $-0.30^{m}$ & 0.06 \\
\hline NGC 104 & 6.03 & -72.08 & 305.89 & -44.89 & $4.50 \pm 0.45^{d}$ & $-48.00 \pm 10.00^{k}$ & ${ }^{k} \quad 5.25 \pm 0.04$ & $-2.52 \pm 0.04$ & -0.71 & 0.36 \\
\hline NGC 5927 & 232.00 & -50.68 & 326.60 & 4.86 & $8.16 \pm 0.27^{e}$ & $-99.00 \pm 11.00^{e}$ & $e-5.05 \pm 0.04$ & $-3.23 \pm 0.04$ & -0.32 & 0.42 \\
\hline NGC 6139 & 246.92 & -38.85 & 342.36 & 6.94 & $9.80 \pm 0.83^{e}$ & $24.41 \pm 0.95^{e}$ & $-6.16 \pm 0.05$ & $-2.67 \pm 0.04$ & -1.42 & 0.15 \\
\hline NGC 6144 & 246.81 & -26.02 & 351.93 & 15.70 & $8.90 \pm 0.89^{d}$ & $195.75 \pm 0.74^{e}$ & $-1.76 \pm 0.04$ & $-2.64 \pm 0.04$ & -2.04 & 0.94 \\
\hline NGC 6171 & 248.13 & -13.06 & 3.37 & 23.01 & $6.03 \pm 0.31^{e}$ & $-34.68 \pm 0.19^{e}$ & $-1.94 \pm 0.04$ & $-5.95 \pm 0.04$ & -1.00 & 0.56 \\
\hline NGC 6235 & 253.36 & -22.17 & 358.92 & 13.52 & $13.52 \pm 1.35^{e}$ & $126.68 \pm 0.33^{e}$ & $-3.94 \pm 0.04$ & $-7.56 \pm 0.04$ & -1.37 & 0.33 \\
\hline NGC 6293 & 257.55 & -26.58 & 357.62 & 7.83 & $9.23 \pm 0.70^{e}$ & $-143.66 \pm 0.39^{e}$ & $0.82 \pm 0.04$ & $-4.31 \pm 0.04$ & -1.92 & 0.05 \\
\hline NGC 6304* & 258.63 & -29.46 & 355.83 & 5.38 & $6.28 \pm 0.11^{f}$ & $-108.62 \pm 0.39^{e}$ & $-4.01 \pm 0.06$ & $-1.03 \pm 0.05$ & $-0.45^{f}$ & 0.21 \\
\hline NGC $6316^{\star}$ & 259.16 & -28.14 & 357.18 & 5.76 & $11.60 \pm 1.16^{e}$ & $92.20 \pm 0.60^{i}$ & $-4.86 \pm 0.06$ & $-4.61 \pm 0.06$ & -0.46 & 0.17 \\
\hline NGC $6325^{\star}$ & 259.50 & -23.77 & 0.97 & 8.00 & $7.80 \pm 0.78^{d}$ & $29.54 \pm 0.58^{e}$ & $-8.41 \pm 0.04$ & $-8.90 \pm 0.05$ & -1.30 & 0.03 \\
\hline NGC 6333 & 259.80 & -18.52 & 5.54 & 10.70 & $8.40 \pm 0.84^{e}$ & $310.75 \pm 2.12^{e}$ & $-2.20 \pm 0.04$ & $-3.21 \pm 0.04$ & -1.70 & 0.45 \\
\hline NGC $6342^{\star}$ & 260.29 & -19.58 & 4.90 & 9.73 & $8.43 \pm 0.84^{e}$ & $116.56 \pm 0.74^{e}$ & $-2.94 \pm 0.05$ & $-7.08 \pm 0.05$ & $-0.60^{m}$ & 0.05 \\
\hline NGC 6352 & 261.37 & -48.43 & 341.42 & -7.17 & $5.89 \pm 0.58^{e}$ & $-123.70 \pm 0.30^{i}$ & $-2.19 \pm 0.04$ & $-4.42 \pm 0.04$ & $-0.48^{f}$ & 0.83 \\
\hline NGC $6355^{\star}$ & 260.99 & -26.36 & 359.58 & 5.43 & $8.70 \pm 0.87^{e}$ & $-210.30 \pm 0.40^{i}$ & $-4.66 \pm 0.06$ & $-0.51 \pm 0.05$ & -1.46 & 0.05 \\
\hline NGC 6356 & 260.89 & -17.82 & 6.72 & 10.22 & $15.10 \pm 1.51^{d}$ & $38.93 \pm 1.88^{e}$ & $-3.77 \pm 0.04$ & $-3.37 \pm 0.04$ & -0.51 & 0.24 \\
\hline NGC $6362^{\star}$ & 262.97 & -67.05 & 325.55 & -17.57 & $7.69 \pm 0.13^{f}$ & $-14.58 \pm 0.18^{e}$ & $-5.47 \pm 0.05$ & $-4.72 \pm 0.05$ & $-1.07^{f}$ & 1.13 \\
\hline NGC 6366 & 261.93 & -5.08 & 18.41 & 16.04 & $3.66 \pm 0.20^{e}$ & $-118.30 \pm 0.30^{i}$ & $-0.38 \pm 0.04$ & $-5.13 \pm 0.04$ & -0.59 & 2.17 \\
\hline NGC 6380 & 263.62 & -39.07 & 350.18 & -3.42 & $9.80 \pm 0.98^{e}$ & $-6.54 \pm 1.48^{e}$ & $-2.10 \pm 0.04$ & $-3.19 \pm 0.04$ & -0.44 & 0.34 \\
\hline NGC 6388 & 264.08 & -44.73 & 345.56 & -6.74 & $10.74 \pm 0.12^{e}$ & $82.85 \pm 0.48^{e}$ & $-1.35 \pm 0.04$ & $-2.71 \pm 0.04$ & -0.51 & 0.12 \\
\hline NGC $6528^{\star}$ & 271.20 & -30.05 & 1.14 & -4.17 & $7.70 \pm 0.77$ & $185.00 \pm 10.00^{k}$ & $k-2.21 \pm 0.07$ & $-5.55 \pm 0.07$ & $-0.17^{m}$ & 0.13 \\
\hline NGC 6535 & 270.96 & -0.29 & 27.18 & 10.44 & $6.50 \pm 0.65^{e}$ & $-214.85 \pm 0.46^{e}$ & $-4.21 \pm 0.04$ & $-2.95 \pm 0.04$ & -1.79 & 0.36 \\
\hline NGC $6539^{\star}$ & 271.21 & -7.58 & 20.80 & 6.78 & $7.85 \pm 0.66^{e}$ & $31.30 \pm 0.40^{i}$ & $-6.89 \pm 0.05$ & $-3.54 \pm 0.05$ & $-0.79^{m}$ & 0.38 \\
\hline NGC $6540^{\star}$ & 271.53 & -27.76 & 3.29 & -3.31 & $5.20 \pm 0.52^{e}$ & $-17.98 \pm 0.84^{e}$ & $-3.68 \pm 0.05$ & $-2.80 \pm 0.06$ & $-0.19^{m}$ & 0.03 \\
\hline NGC 6541 & 272.00 & -43.71 & 349.29 & -11.18 & $7.95 \pm 0.37^{e}$ & $-163.97 \pm 0.46^{e}$ & $0.28 \pm 0.04$ & $-8.77 \pm 0.04$ & -1.80 & 0.18 \\
\hline NGC 6544 & 271.83 & -24.99 & 5.84 & -2.20 & $3.00 \pm 0.30^{d}$ & $-38.12 \pm 0.76^{e}$ & $-2.33 \pm 0.04$ & $-18.56 \pm 0.04$ & -1.37 & 0.05 \\
\hline NGC 6553 & 272.32 & -25.90 & 5.25 & -3.02 & $6.75 \pm 0.22^{e}$ & $0.72 \pm 0.40^{e}$ & $0.26 \pm 0.04$ & $-0.41 \pm 0.04$ & -0.12 & 0.53 \\
\hline NGC $6558^{\star}$ & 272.57 & -31.76 & 0.20 & -6.02 & $8.26 \pm 0.53^{h}$ & $-194.45 \pm 2.10^{h}$ & $-1.78 \pm 0.05$ & $-4.12 \pm 0.04$ & $-1.17^{h}$ & 0.03 \\
\hline NGC 6569 & 273.41 & -31.83 & 0.48 & -6.68 & $10.59 \pm 0.80^{e}$ & $-49.83 \pm 0.50^{e}$ & $-4.13 \pm 0.04$ & $-7.26 \pm 0.04$ & -0.73 & 0.35 \\
\hline NGC $6624^{\star}$ & 275.92 & -30.36 & 2.79 & -7.91 & $8.43 \pm 0.11^{f}$ & $54.26 \pm 0.45^{e}$ & $0.10 \pm 0.05$ & $-6.92 \pm 0.05$ & $-0.69^{f}$ & 0.06 \\
\hline NGC $6626^{\star}$ & 276.14 & -24.87 & 7.80 & -5.58 & $5.34 \pm 0.17^{g}$ & $11.11 \pm 0.60^{e}$ & $-0.31 \pm 0.06$ & $-8.93 \pm 0.06$ & $-1.00^{m}$ & 0.24 \\
\hline NGC $6637^{\star}$ & 277.85 & -32.35 & 1.72 & -10.27 & $8.80 \pm 0.88$ & $46.63 \pm 1.45^{e}$ & $-5.11 \pm 0.06$ & $-5.82 \pm 0.06$ & $-0.77^{m}$ & 0.33 \\
\hline NGC $6638^{\star}$ & 277.73 & -25.49 & 7.90 & -7.15 & $10.32 \pm 1.03^{e}$ & $8.63 \pm 2.00^{e}$ & $-2.55 \pm 0.05$ & $-4.08 \pm 0.05$ & -0.89 & 0.22 \\
\hline NGC $6642^{\star}$ & 277.97 & -23.48 & 9.81 & -6.44 & $8.10 \pm 0.81^{d}$ & $-33.23 \pm 1.13^{e}$ & $-0.23 \pm 0.05$ & $-3.88 \pm 0.05$ & -1.03 & 0.10 \\
\hline NGC $6652^{\star}$ & 278.94 & -32.99 & 1.53 & -11.38 & $9.51 \pm 0.12^{f}$ & $-99.04 \pm 0.51^{e}$ & $-5.47 \pm 0.05$ & $-4.20 \pm 0.05$ & $-0.85^{f}$ & 0.10 \\
\hline NGC 6656 & 138.69 & -49.23 & 9.89 & -7.55 & $3.20 \pm 0.32^{d}$ & $-152.00 \pm 25.00^{k}$ & $k \quad 9.80 \pm 0.04$ & $-5.56 \pm 0.04$ & -1.92 & 1.33 \\
\hline NGC 6681 & 283.26 & -8.70 & 2.85 & -12.51 & $9.31 \pm 0.17^{e}$ & $216.62 \pm 0.84^{e}$ & $1.39 \pm 0.04$ & $-4.72 \pm 0.04$ & -1.55 & 0.03 \\
\hline NGC 6712 & 283.27 & -8.70 & 25.35 & -4.39 & $6.95 \pm 0.39^{e}$ & $-107.45 \pm 0.29^{e}$ & $3.32 \pm 0.04$ & $-4.38 \pm 0.04$ & -0.97 & 0.76 \\
\hline NGC $6717^{\star}$ & 283.78 & -22.70 & 12.88 & -10.90 & $7.14 \pm 0.10^{f}$ & $32.45 \pm 1.44^{e}$ & $-3.16 \pm 0.05$ & $-4.92 \pm 0.05$ & $-1.26^{f}$ & 0.08 \\
\hline NGC $6723^{\star}$ & 284.89 & -36.63 & 0.07 & -17.30 & $8.17 \pm 0.11^{f}$ & $-94.18 \pm 0.26^{e}$ & $0.99 \pm 0.05$ & $-2.45 \pm 0.05$ & $-1.10^{f}$ & 0.83 \\
\hline NGC 6752 & 287.71 & -59.98 & 336.49 & -25.63 & $4.25 \pm 0.09^{e}$ & $-26.28 \pm 0.16^{e}$ & $-3.17 \pm 0.04$ & $-4.01 \pm 0.04$ & -1.41 & 0.17 \\
\hline NGC 6760 & 287.80 & 1.03 & 36.11 & -3.92 & $7.95 \pm 0.50^{e}$ & $-0.42 \pm 1.63^{e}$ & $-1.11 \pm 0.04$ & $-3.59 \pm 0.04$ & -0.41 & 0.34 \\
\hline NGC 6809 & 295.00 & -30.97 & 8.79 & -23.27 & $5.30 \pm 0.20^{e}$ & $174.40 \pm 0.24^{e}$ & $-3.40 \pm 0.04$ & $-9.26 \pm 0.04$ & -1.87 & 1.80 \\
\hline NGC 6838 & 298.44 & 18.78 & 56.75 & -4.56 & $4.00 \pm 0.20^{d}$ & $-42.00 \pm 18.00^{k}$ & $k-3.38 \pm 0.04$ & $-2.65 \pm 0.04$ & -0.63 & 0.63 \\
\hline Palomar 11 & 296.31 & -8.01 & 31.80 & -15.57 & $14.30 \pm 1.40^{e}$ & $-67.64 \pm 0.76^{e}$ & $-1.79 \pm 0.05$ & $-4.94 \pm 0.05$ & -0.35 & 1.19 \\
\hline Palomar $6^{\star}$ & 265.93 & -26.22 & 2.10 & 1.78 & $5.80 \pm 0.58^{e}$ & $177.00 \pm 1.00^{i}$ & $-9.11 \pm 0.06$ & $-5.27 \pm 0.06$ & $-1.00^{m}$ & 0.66 \\
\hline
\end{tabular}


Table 1 - continued

\begin{tabular}{|c|c|c|c|c|c|c|c|c|c|c|}
\hline Cluster & $\begin{array}{l}\alpha_{2000} \\
(\mathrm{deg})\end{array}$ & $\begin{array}{l}\delta_{2000} \\
(\operatorname{deg})\end{array}$ & $\begin{array}{c}1 \\
(\mathrm{deg})\end{array}$ & $\begin{array}{c}\mathrm{b} \\
(\mathrm{deg})\end{array}$ & $\begin{array}{c}\mathrm{d}_{\odot} \\
(\mathrm{kpc})\end{array}$ & $\begin{array}{c}V_{\mathrm{r}} \\
\left(\mathrm{km} \mathrm{s}^{-1}\right)\end{array}$ & $\begin{array}{c}\mu_{\alpha}^{*} \\
\left(\operatorname{mas~yr}^{-1}\right)\end{array}$ & $\begin{array}{c}\mu_{\delta} \\
\left(\operatorname{mas~yr}^{-1}\right)\end{array}$ & $\begin{array}{c}{[\mathrm{Fe} / \mathrm{H}]^{\dagger}} \\
(\mathrm{dex})\end{array}$ & $\begin{array}{c}\mathrm{R}_{c}^{\ddagger} \\
(\operatorname{arcmin})\end{array}$ \\
\hline Palomar 8 & 280.37 & -19.82 & 14.10 & -6.78 & $12.80 \pm 1.28^{e}$ & $-41.14 \pm 1.81^{e}$ & $-2.04 \pm 0.04$ & $-5.64 \pm 0.04$ & -0.34 & 0.56 \\
\hline Terzan $1^{\star}$ & 263.95 & -30.47 & 357.57 & 1.00 & $6.70 \pm 0.67$ & $63.00 \pm 0.50^{i}$ & $-3.02 \pm 0.05$ & $-4.49 \pm 0.04$ & $-1.26^{m}$ & 0.04 \\
\hline Terzan 12 & 273.06 & -22.74 & 8.36 & -2.10 & $3.40 \pm 0.34^{e}$ & $94.77 \pm 0.97^{e}$ & $-6.07 \pm 0.07$ & $-2.63 \pm 0.07$ & -0.47 & 0.83 \\
\hline Terzan $2^{\star}$ & 261.89 & -30.80 & 356.32 & 2.30 & $7.50 \pm 0.75^{d}$ & $144.60 \pm 0.80^{d}$ & $-2.21 \pm 0.06$ & $-6.16 \pm 0.05$ & -0.42 & 0.03 \\
\hline Terzan $3^{\star}$ & 247.17 & -35.35 & 345.08 & 9.19 & $8.20 \pm 0.82^{d}$ & $-135.76 \pm 0.57^{e}$ & $-5.61 \pm 0.04$ & $-1.69 \pm 0.04$ & -0.96 & 1.18 \\
\hline Terzan $6^{\star}$ & 267.69 & -31.28 & 358.57 & -2.16 & $6.80 \pm 0.68^{d}$ & $137.15 \pm 1.70^{e}$ & $-5.70 \pm 0.11$ & $-7.20 \pm 0.11$ & -0.53 & 0.05 \\
\hline Terzan $9^{\star}$ & 270.41 & -26.84 & 3.61 & -1.99 & $7.10 \pm 0.71^{d}$ & $71.40 \pm 0.40^{i}$ & $-2.19 \pm 0.06$ & $-7.47 \pm 0.05$ & -1.08 & 0.03 \\
\hline Ton $2^{\star}$ & 264.04 & -38.55 & 350.80 & -3.42 & $6.40 \pm 0.64^{e}$ & $-172.70 \pm 0.30^{i}$ & $-5.92 \pm 0.05$ & $-0.55 \pm 0.05$ & -0.26 & 0.54 \\
\hline
\end{tabular}

Notes. References. ${ }^{a}$ Ortolani et al. (2019a) ; ${ }^{b}$ Ortolani et al. (2019b); ${ }^{c}$ Kerber et al. (2019); ${ }^{d}$ Harris (1996, updated in 2010), ${ }^{e}$ Baumgardt et al. (2019); ${ }^{f}$ Oliveira et al. (2019, in preparation); ${ }^{g}$ Kerber et al. (2018); ${ }^{h}$ Barbuy et al. (2018b); ${ }^{i}$ Vásquez et al. (2018); ${ }^{j}$ Barbuy et al. (2016); ${ }^{k}$ Dias et al. (2016); ${ }^{l}$ Barbuy et al. (2014); ${ }^{m}$ Bica et al (2016, their table 3). ${ }^{\dagger}$ Metallicities with no reference come from the website by Bruno Dias (http://www.sc.eso.org/ bdias/catalogues.html). ${ }^{\ddagger} R_{c}$ taken from H10.
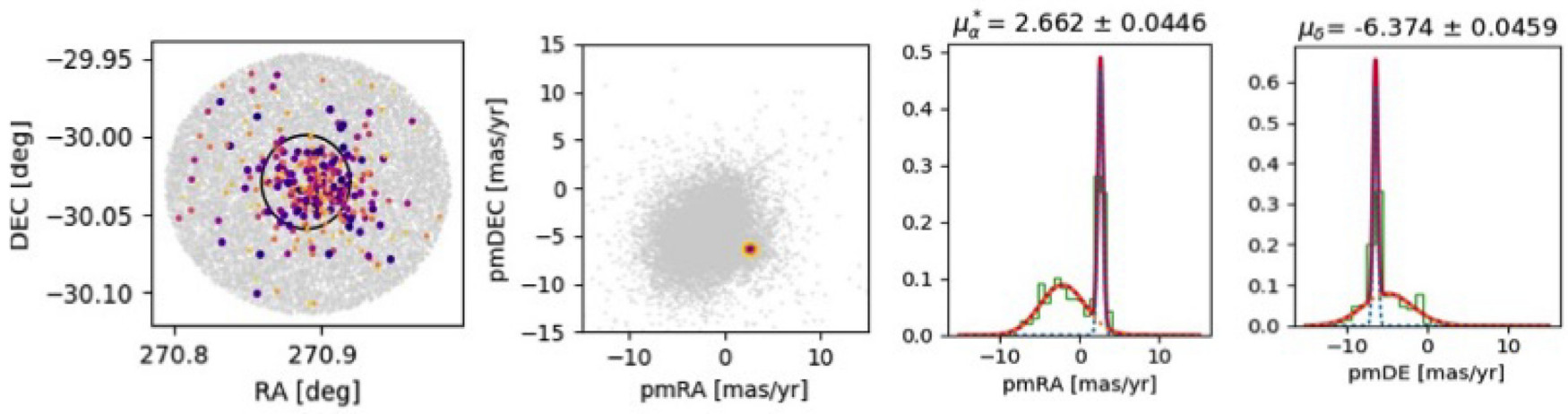

Figure 2. Example of proper motion data from Gaia DR2 for NGC 6522, and cluster member selection. First panel: core radius containing the field studied; second panel: location of the cluster in the vector-point diagram; third and fourth panels: Gaussian of RA and Dec. proper motions, respectively.

7, and in column 8 is given the distance compiled by Bica et al. (2006).

The GCs with the largest difference in distance determinations in the literature are Djorg 1, Djorg 2, ESO452SC11, NGC 5927, NGC 6235, NGC 6256, NGC 6316, NGC 6380, NGC 6388, NGC 6401, NGC 6540, NGC 6553, NGC 6638, Palomar 6, Terzan 4, Terzan 5, and Terzan 9.

In order to have a reliable statistical estimate of the uncertainty in distances, we first compared distances from Bica et al. (2006) with those from Valenti et al. $(2007,2010)$ for 17 bulge clusters in common. These two samples are independent because the first is mostly optical-based and the other is near-infrared-based, with different reddening dependence and different isochrone sets. From the whole sample, we have an uncertainty $\sigma=1.3 \mathrm{kpc}$, but by removing two outliers with errors larger than $2 \mathrm{kpc}$, which clearly have some specific problems, the uncertainty goes down to $\sigma$ $=0.99 \mathrm{kpc}$. A second check comparing our adopted distances with respect to Baumgardt et al. (2019) and removing two outliers with differences larger than $4 \mathrm{kpc}$, this results in a standard deviation of $0.4 \mathrm{kpc}$. From these two comparisons, we adopt a mean standard deviation of $0.7 \mathrm{kpc}$.

Note that Hilker et al. (2019) report an error on their proper motion distances of 8 per cent at the distance of $7 \mathrm{kpc}$, which gives an error of $0.6 \mathrm{kpc}$ at the typical distance of the bulge clusters of
$7 \mathrm{kpc}$. This is very consistent with the suggested $0.7 \mathrm{kpc}$ standard deviation.

\section{THE GALACTIC POTENTIAL}

In order to construct the orbits of the GCs, in this study we employ a non-axisymmetric model for the Galactic gravitational potential, which is built from an axisymmetric background that includes a Sérsic bulge, an exponential disc made by the superposition of three Miyamoto-Nagai potentials (Miyamoto \& Nagai 1975), following the recipe given by Smith et al. (2015), and a Navarro-FrenkWhite density profile (Navarro, Frenk \& White 1997) to model the dark matter halo, having a circular velocity $V_{0}=241 \mathrm{~km} \mathrm{~s}^{-1}$ at $R_{0}=8.2 \mathrm{kpc}$ (Bland-Hawthorn \& Gerhard 2016). Even though the mass of the dark matter halo is twice larger than usual halo masses of $1.0-1.5 \times 10^{12} \mathrm{M}_{\odot}$, with the scale radius we assumed, the rotation curve inside $30 \mathrm{kpc}$ of our model is in agreement with Bland-Hawthorn \& Gerhard (2016), as shown in Fig. 3. We also adopted a less massive DM halo, also reducing the scale radius, and no significant differences are found.

For the Galactic bar, we used a triaxial Ferrer's ellipsoid, where all the mass from the bulge component is converted into a bar. For the bar potential, we consider a total bar mass of $1.2 \times 10^{10} \mathrm{M}_{\odot}$, an angle of $25^{\circ}$ with the Sun-major axis of the bar, a major axis extension of $3.5 \mathrm{kpc}$, and a gradient of the bar pattern speed values 
Table 2. Globular cluster distances from the literature.

\begin{tabular}{|c|c|c|c|c|c|c|c|}
\hline Cluster & $\begin{array}{l}\text { Adopted } \\
(\mathrm{kpc})\end{array}$ & $\begin{array}{l}\mathrm{H} 10 \\
(\mathrm{kpc})\end{array}$ & $\begin{array}{c}\text { Baumgardt }+19 \\
(\mathrm{kpc})\end{array}$ & $\begin{array}{c}\text { Valenti }+07,10 \\
(\mathrm{kpc})\end{array}$ & $\begin{array}{l}\text { Gaia }(1 / \mathrm{plx}) \\
\quad(\mathrm{kpc})\end{array}$ & $\begin{array}{l}\text { Our group } \\
\quad(\mathrm{kpc})\end{array}$ & $\begin{array}{l}\text { Bica }+06 \\
\quad(\mathrm{kpc})\end{array}$ \\
\hline BH 261 & $6.50 \pm 0.65$ & 6.50 & 6.50 & - & - & - & - \\
\hline Djorg $1^{*}$ & $9.30 \pm 0.50$ & 13.70 & 13.70 & 13.50 & - & 9.30 & 8.80 \\
\hline Djorg $2^{*}$ & $8.75 \pm 0.20$ & 6.30 & 6.30 & 7.0 & 9.60 & 8.75 & 6.70 \\
\hline ESO452SC11* & $6.50 \pm 0.65$ & 8.30 & 6.50 & - & - & - & 7.8 \\
\hline HP 1 & $6.59 \pm 0.16$ & 8.20 & 6.80 & 6.80 & 50.0 & 6.59 & 7.40 \\
\hline Liller 1 & $8.20 \pm 0.82$ & 8.20 & 8.10 & 7.90 & - & 7.60 & 10.50 \\
\hline Lynga 7 & $8.0 \pm 0.80$ & 8.0 & 8.0 & - & - & - & 7.2 \\
\hline Mercer 5 & $5.50 \pm 0.55$ & - & 5.50 & - & - & - & - \\
\hline NGC 104 & $4.50 \pm 0.45$ & 4.50 & 4.43 & - & 5.10 & - & 4.50 \\
\hline NGC 5927* & $8.16 \pm 0.27$ & 7.70 & 8.16 & - & 10.04 & - & 7.60 \\
\hline NGC 6139 & $9.80 \pm 0.83$ & 10.10 & 9.8 & - & - & - & 10.1 \\
\hline NGC 6144 & $8.90 \pm 0.89$ & 8.90 & 8.90 & - & 14.97 & - & 10.30 \\
\hline NGC 6171 & $6.03 \pm 0.31$ & 6.40 & 6.03 & - & 6.76 & - & 6.40 \\
\hline NGC $6235^{*}$ & $13.52 \pm 1.35$ & 11.50 & 13.52 & - & 16.18 & - & 10.00 \\
\hline NGC $6256^{*}$ & $6.40 \pm 0.64$ & 10.30 & 6.40 & 9.10 & - & 6.4 & 6.60 \\
\hline NGC 6266 & $6.41 \pm 0.12$ & 6.80 & 6.41 & 6.6 & 4.57 & - & 6.90 \\
\hline NGC 6273 & $8.27 \pm 0.41$ & 8.80 & 8.27 & 8.20 & 10.82 & - & 8.70 \\
\hline NGC 6284 & $15.3 \pm 1.53$ & 15.3 & 15.14 & - & 20.04 & - & 14.70 \\
\hline NGC 6287 & $9.40 \pm 0.94$ & 9.40 & 9.40 & - & 9.31 & - & 8.50 \\
\hline NGC 6293 & $9.23 \pm 0.70$ & 9.50 & 9.23 & 10.50 & 14.37 & - & 8.80 \\
\hline NGC 6304 & $6.28 \pm 0.11$ & 5.9 & 5.77 & 6.0 & 9.29 & 6.19 & 6.10 \\
\hline NGC 6316* & $11.60 \pm 1.16$ & 10.40 & 11.60 & 11.6 & 15.17 & - & 11.00 \\
\hline NGC 6325 & $7.80 \pm 0.78$ & 7.80 & 7.80 & - & 6.99 & 6.90 & 9.60 \\
\hline NGC 6333 & $8.40 \pm 0.84$ & 7.90 & 8.40 & - & 10.70 & - & 8.20 \\
\hline NGC 6342 & $8.43 \pm 0.84$ & 8.50 & 8.43 & 8.40 & 10.28 & - & 8.60 \\
\hline NGC 6352 & $5.89 \pm 0.58$ & 5.60 & 5.89 & - & 6.48 & - & 5.70 \\
\hline NGC 6355 & $8.70 \pm 0.87$ & 9.20 & 8.70 & 9.0 & - & 8.80 & 7.20 \\
\hline NGC 6356 & $15.10 \pm 1.51$ & 15.10 & 15.10 & - & 12.64 & 15 & 15.20 \\
\hline NGC 6362 & $7.69 \pm 0.13$ & 7.6 & 7.36 & - & 10.27 & 7.71 & 8.10 \\
\hline NGC 6366 & $3.66 \pm 0.20$ & 3.5 & 3.66 & - & 4.36 & - & 3.60 \\
\hline NGC 6380* & $9.80 \pm 0.98$ & 10.90 & 9.80 & 9.20 & 9.86 & 9.8 & 10.70 \\
\hline NGC 6388* & $10.74 \pm 0.12$ & 9.90 & 10.74 & 11.9 & 20.74 & - & 11.50 \\
\hline NGC 6401* & $7.7 \pm 0.77$ & 10.6 & 7.7 & 7.7 & 8.65 & 12.0 & 7.70 \\
\hline NGC 6402 & $9.31 \pm 0.44$ & 9.30 & 9.31 & - & 18.66 & - & 8.90 \\
\hline NGC 6440 & $8.24 \pm 0.82$ & 8.5 & 8.24 & 8.20 & 10.43 & 8.47 & 8.40 \\
\hline NGC 6441 & $11.83 \pm 0.14$ & 11.60 & 11.83 & 13.5 & 24.81 & - & 11.20 \\
\hline NGC 6453 & $11.60 \pm 1.16$ & 11.60 & 11.60 & 10.7 & 23.53 & 8.5 & 11.20 \\
\hline NGC 6496 & $11.30 \pm 1.13$ & 11.30 & 11.30 & - & 12.45 & - & 6.60 \\
\hline NGC 6517 & $10.60 \pm 1.06$ & 10.60 & 10.60 & - & 46.08 & - & 10.80 \\
\hline NGC 6522 & $7.40 \pm 0.19$ & 7.70 & 8 & 7.40 & 14.35 & 7.40 & 7.80 \\
\hline NGC 6528 & $7.70 \pm 0.77$ & 7.90 & 7.45 & 7.50 & 13.40 & 7.50 & 9.10 \\
\hline NGC 6535 & $6.50 \pm 0.65$ & 6.80 & 6.50 & - & 7.73 & - & 6.70 \\
\hline NGC 6539 & $7.85 \pm 0.66$ & 7.8 & 7.85 & 8.4 & 15.87 & - & 8.40 \\
\hline NGC $6540^{*}$ & $5.20 \pm 0.52$ & 5.30 & 5.20 & 5.20 & - & 3.50 & 3.70 \\
\hline NGC 6541 & $7.95 \pm 0.37$ & 7.5 & 7.95 & - & 8.78 & - & 7.00 \\
\hline NGC 6544 & $3.0 \pm 0.30$ & 3.0 & 2.60 & 2.80 & 3.02 & - & 2.60 \\
\hline NGC 6553* & $6.75 \pm 0.22$ & 6.0 & 6.75 & 4.90 & - & 5.20 & 5.60 \\
\hline NGC 6558 & $8.26 \pm 0.53$ & 7.40 & 7.20 & - & - & 8.26 & 7.40 \\
\hline NGC 6569 & $10.59 \pm 0.80$ & 10.90 & 10.59 & 12.0 & - & - & 8.7 \\
\hline NGC 6624 & $8.43 \pm 0.11$ & 7.9 & 7.19 & 8.4 & 7.26 & 8.32 & 8.00 \\
\hline NGC 6626 & $5.34 \pm 0.17$ & 5.5 & 5.43 & - & 6.81 & 5.34 & 5.70 \\
\hline NGC 6637 & $8.80 \pm 0.88$ & 8.80 & 8.80 & 9.40 & 13.40 & 8.85 & 8.60 \\
\hline NGC 6638* & $10.32 \pm 1.03$ & 9.40 & 10.32 & 13.5 & - & - & 8.40 \\
\hline NGC 6642 & $8.10 \pm 0.81$ & 8.10 & 8.05 & 7.0 & - & - & 7.70 \\
\hline NGC 6652 & $9.51 \pm 0.12$ & 10 & 10 & 6.8 & 9.09 & 9.44 & 9.60 \\
\hline NGC 6656 & $3.20 \pm 0.32$ & 3.20 & 3.23 & 7.90 & 3.84 & - & 3.20 \\
\hline NGC 6681 & $9.31 \pm 0.17$ & 9.0 & 9.31 & - & 9.12 & - & - \\
\hline NGC 6712 & $6.95 \pm 0.39$ & 6.90 & 6.12 & - & - & - & 6.9 \\
\hline NGC 6717 & $7.14 \pm 0.10$ & 7.10 & 7.10 & - & 8.0 & 7.15 & 7.40 \\
\hline NGC 6723 & $8.17 \pm 0.11$ & 8.70 & 8.30 & - & 13.33 & 8.05 & 8.80 \\
\hline NGC 6752 & $4.25 \pm 0.09$ & 4.0 & 4.25 & - & 4.32 & 4.36 & 4.0 \\
\hline NGC 6760 & $7.95 \pm 0.50$ & 7.40 & 7.95 & - & - & - & 7.40 \\
\hline NGC 6809 & $5.30 \pm 0.20$ & 5.40 & 5.30 & - & 5.86 & - & 5.40 \\
\hline
\end{tabular}


Table 2 - continued

\begin{tabular}{lccccccc}
\hline Cluster & $\begin{array}{c}\text { Adopted } \\
(\mathrm{kpc})\end{array}$ & $\begin{array}{c}\mathrm{H} 10 \\
(\mathrm{kpc})\end{array}$ & $\begin{array}{c}\text { Baumgardt }+19 \\
(\mathrm{kpc})\end{array}$ & $\begin{array}{c}\text { Valenti }+07,10 \\
(\mathrm{kpc})\end{array}$ & $\begin{array}{c}\text { Gaia }(1 / \mathrm{plx}) \\
(\mathrm{kpc})\end{array}$ & $\begin{array}{c}\text { Our group } \\
(\mathrm{kpc})\end{array}$ & $\begin{array}{c}\text { Bica }+06 \\
(\mathrm{kpc})\end{array}$ \\
\hline NGC 6838 & $4.0 \pm 0.20$ & 4.0 & 3.99 & - & 4.44 & - & 3.90 \\
Palomar 11 & $14.30 \pm 1.40$ & 13.4 & 14.30 & - & - & - & 12.9 \\
Palomar 6* & $5.80 \pm 0.58$ & 5.80 & 5.80 & - & - & 8.90 & 7.30 \\
Palomar 7 & $5.39 \pm 0.51$ & 5.40 & 5.38 & - & - & - & 5.4 \\
Palomar 8 & $12.80 \pm 1.28$ & 12.80 & 12.80 & - & - & - & 12.9 \\
Terzan 1 & $6.70 \pm 0.67$ & 6.70 & 6.7 & 9.10 & - & 5.20 & 6.20 \\
Terzan 10 & $10.3 \pm 1.0$ & 5.8 & 5.8 & 6.6 & - & 10.30 & 5.70 \\
Terzan 12 & $3.40 \pm 0.34$ & 4.80 & 3.40 & - & - & - & 4.80 \\
Terzan 2 & $7.50 \pm 0.75$ & 7.50 & 7.50 & 8.20 & - & 7.70 & 8.70 \\
Terzan 3 & $8.20 \pm 0.82$ & 8.20 & 8.10 & - & - & 6.50 & 7.50 \\
Terzan 4* & $6.70 \pm 0.67$ & 7.20 & 6.70 & - & - & 8.0 & 9.10 \\
Terzan 5* & $5.50 \pm 0.51$ & 6.90 & 5.50 & 10.50 & - & 5.50 & 7.60 \\
Terzan 6 & $6.80 \pm 0.68$ & 6.80 & 6.70 & 6.0 & - & 7.0 & 9.50 \\
Terzan 9* & $7.10 \pm 0.71$ & 7.10 & 7.10 & 11.60 & - & 4.90 & 7.70 \\
Ton 2 & $6.40 \pm 0.64$ & 8.20 & 6.40 & - & - & 6.40 & 8.10 \\
\hline
\end{tabular}

*GCs with the largest difference in distance determinations in the literature.

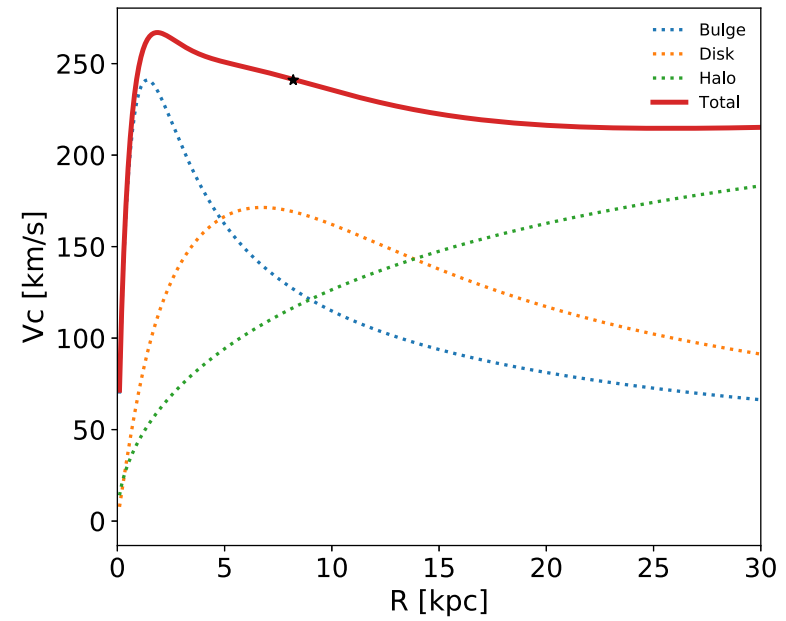

Figure 3. Total circular velocity curve and the contribution of each component of axisymmetric Galactic mass model. The black star shows the velocity at the Sun position.

assumed to be $\Omega_{b}=40,45$, and $50 \mathrm{~km} \mathrm{~s}^{-1} \mathrm{kpc}^{-1}$. We keep the same bar extension in all cases, for any of the bar pattern speed values. Table 3 gives the parameters used for our Galactic model.

\section{PROPERTIES OF THE GLOBULAR CLUSTER ORB I T S}

To construct the GC orbits through the Galactic potential, we have employed the Shampine-Gordon integration scheme that is implemented in the NIGO tool (Numerical Integrator of Galactic Orbits - Rossi 2015a).

We integrated the orbits for the present cluster sample forward in time for $10 \mathrm{Gyr}$. Using the observational parameters of the GCs given in Table 1, we computed the initial state vector of the clusters assuming the Sun's Galactocentric distance $R_{0}=8.2 \mathrm{kpc}$ and a circular velocity $V_{0}=241 \mathrm{~km} \mathrm{~s}^{-1}$ (e.g. Bland-Hawthorn \& Gerhard 2016, and references therein). The velocity components of the Sun with respect to the local standard of rest are $(U, V$, $W)_{\odot}=(11.1,12.24,7.25) \mathrm{km} \mathrm{s}^{-1}$ (Schönrich, Binney \& Dehnen 2010). The velocity components of the cluster in the heliocentric
Table 3. Parameters of the adopted Galactic mass model.

\begin{tabular}{|c|c|c|}
\hline Parameter & Value & Reference \\
\hline \multicolumn{3}{|c|}{ Axisymmetric components } \\
\hline \multicolumn{3}{|c|}{ Bulge } \\
\hline$M_{\mathrm{b}, \text { tot }}$ & $1.2 \times 10^{10} \mathrm{M}_{\odot}$ & 1,2 \\
\hline$R_{\mathrm{e}}$ & $0.87 \mathrm{kpc}$ & - \\
\hline$n$ & 1 & 3 \\
\hline \multicolumn{3}{|c|}{ Disc } \\
\hline$M_{\mathrm{d}, 1}$ & $1.07 \times 10^{11} \mathrm{M}_{\odot}$ & - \\
\hline$a_{\mathrm{d}, 1}$ & $5.97 \mathrm{kpc}$ & - \\
\hline$M_{\mathrm{d}, 2}$ & $-7.09 \times 10^{10} \mathrm{M}_{\odot}$ & - \\
\hline$a_{\mathrm{d}, 2}$ & $12.99 \mathrm{kpc}$ & - \\
\hline$M_{\mathrm{d}, 3}$ & $1.2 \times 10^{10} \mathrm{M}_{\odot}$ & - \\
\hline$a_{\mathrm{d}, 3}$ & $2.04 \mathrm{kpc}$ & - \\
\hline$b_{\mathrm{d}}$ & $0.25 \mathrm{kpc}$ & - \\
\hline \multicolumn{3}{|c|}{ Halo } \\
\hline$M_{\mathrm{h}}$ & $3.0 \times 10^{12} \mathrm{M}_{\odot}$ & 4 \\
\hline$a_{\mathrm{h}}$ & $57.0 \mathrm{kpc}$ & 4 \\
\hline \multicolumn{3}{|c|}{ Galactic bar } \\
\hline$M_{\text {bar }}$ & $1.2 \times 10^{10} \mathrm{M}_{\odot}$ & $1,2,5$ \\
\hline$n$ & 2 & 6 \\
\hline$a$ & $3.5 \mathrm{kpc}$ & 9,10 \\
\hline$b$ & $1.4 \mathrm{kpc}$ & 9,10 \\
\hline$c$ & $1.0 \mathrm{kpc}$ & 9,10 \\
\hline$\phi_{\text {bar }}$ & $25^{\circ}$ & $2,7,8$ \\
\hline$\Omega_{\text {bar }}$ & $40,45,50 \mathrm{~km} \mathrm{~s}^{-1} \mathrm{kpc}^{-1}$ & $2,11,12,13$ \\
\hline
\end{tabular}

Note. References. (1) Portail et al. (2015a); (2) BlandHawthorn \& Gerhard (2016); (3) Kent et al. (1991); (4) Irrgang et al. (2013); (5) Weiner \& Sellwood (1999); (6) Pfenniger (1984); (7) Rattenbury et al. (2007); (8) Wegg \& Gerhard (2013); (9) Freudenreich (1998); (10) Gardner \& Flynn (2010); (11) Bissantz, Englmaier \& Gerhard (2003); (12) Portail et al. (2017); (13) Pérez-Villegas et al. (2017).

reference system $U, V$, and $W$ are positive in direction of the Galactic centre, Galactic rotation, and North Galactic Pole, respectively. We defined as inertial Galactocentric frame of reference the righthanded system of coordinates $(x, y, z)$, where the $x$-axis points to the Sun from the Galactic Centre and the $z$-axis points to the North Galactic Pole. We refer to the bar-corotating frame of reference as the right-handed system of coordinates $\left(x_{\mathrm{r}}, y_{\mathrm{r}}, z_{\mathrm{r}}\right)$ that corotates with 

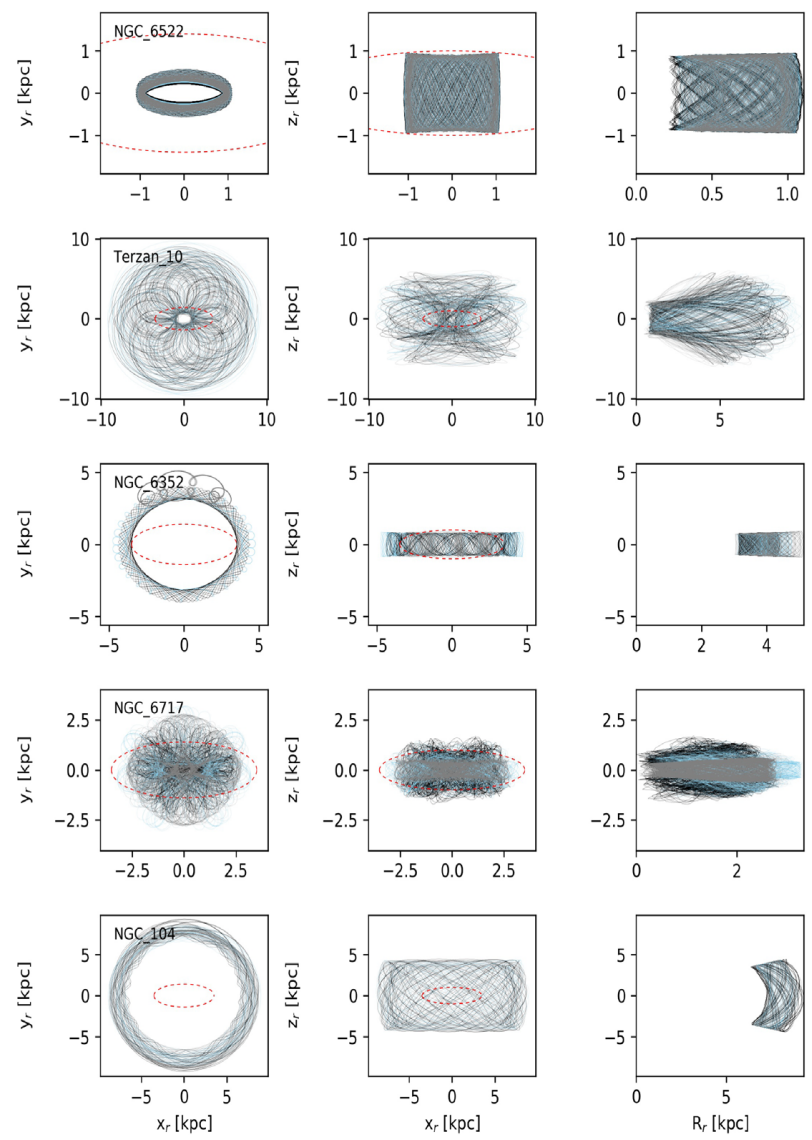

Figure 4. Examples of orbits for the globular clusters. The three columns show $x_{r}-y_{r}, x_{\mathrm{r}}-z_{r}$, and $R_{r}-z_{r}$ projections for orbits with the nonaxisymmetric Galactic potential corotating with the bar. The colours in the left-hand panels are the orbits with different pattern speed of the bar, 40 (black), 45 (blue), and 50 (grey) $\mathrm{km} \mathrm{s}^{-1} \mathrm{kpc}^{-1}$. The dashed red line shows the size of the Galactic bar.

the bar, where the $x_{\mathrm{r}}$-axis is aligned with the bar semimajor axis $a$ and $z_{\mathrm{r}}$ points towards the north Galactic pole.

For each cluster, we generate a set of 1000 initial conditions, where we follow the Monte Carlo technique taking into account the uncertainties on the heliocentric distance, PMs in both directions, and the radial velocity given in Table 1 . The PM uncertainties include the average of the systematic error of 0.035 mas $\mathrm{yr}^{-1}$ reported by the Gaia Collaboration (2018b). These calculations were carried out with the purpose of evaluating how much the orbital properties of the GCs change due to the uncertainties of the observed data. Fig. 4 shows some examples of orbits with the three different bar pattern speed values, in the frame corotating with the bar, for five GCs of our sample: NGC 6522, Terzan 10, NGC 6352, NGC 6717, and NGC 104 (from top to bottom). The initial conditions for them are the central values given in Table 1.

The orbital properties we calculate, in the inertial frame of reference, are the perigalactic distance $r_{\min }$, the apogalactic distance $r_{\max }$, the maximum vertical excursion from the Galactic plane $|z|_{\max }$, and the eccentricity defined as $e=\left(r_{\max }-r_{\min }\right) /\left(r_{\max }+r_{\min }\right)$.

In Table 4, we present the orbital parameters of our sample of GCs, where we use three values of rotational velocity of the bar $\Omega_{b}=40,45$, and $50 \mathrm{~km} \mathrm{~s}^{-1} \mathrm{kpc}^{-1}$. For each bar pattern speed, we give the median values of the perigalactic distance $<r_{\min }>$, apogalactic distance $<r_{\max }>$, the maximum vertical excursion from the Galactic plane $<|z|_{\max }>$, and the orbital eccentricity $<e>$. The errors provided in each column are the standard deviation of the distribution. We see that for the clusters that are confined in the innermost Galaxy, such as HP 1, Djorg 2, Liller 1, and NGC 6325, the effect of the bar pattern speed is almost negligible. On the contrary, for NGC 5927, the perigalactic distance decreases with the pattern speed.

\section{CLASSIFYING GLOBULAR CLUSTERS}

In the literature, GCs have been classified based on their spatial distribution, metallicity, and internal and structural parameters (e.g. Aguilar, Hut \& Ostriker 1988; Minniti 1995; Barbuy, Bica \& Ortolani 1998; Côté 1999; Bica et al. 2016; Pasquato \& Chung 2019). In this section, we used the orbital parameters of the $78 \mathrm{GCs}$, calculated in Section 4, to separate the clusters into different stellar populations. We obtained this classification using unsupervised clustering algorithms.

\subsection{Clustering method}

The identification of GCs that are confined or belong to the bulge/bar component, which is the main aim of this work, is not an easy task because in the innermost part of the Galaxy, all components overlap: the bulge/bar, thick disc, inner halo, and outer halo.

In terms of dynamics, the GCs of each component should share its dynamical properties. Based on this criterion, we employed the orbital parameters (Table 4) to separate the GCs into four Galactic components: bulge/bar, thick disc, inner halo, and outer halo. To do this classification, we employ the GMMs, an unsupervised machine learning algorithm that searches for $K$ Gaussian distributions, which fits better an ND parameter space. Based on Bayes's theorem, the GMM algorithm tries to maximize the expression:

$G(x)=\sum_{i=1}^{K} \phi_{i} \times \mathcal{N}\left(x \mid \mu_{i}, \sigma_{i}\right)$,

where $\mathcal{N}\left(x \mid \mu_{i}, \sigma_{i}\right)$ represents the ith Gaussian distribution with mean $\mu_{i}$ and standard deviation $\sigma_{i}$. The $\vec{x}$ is the parameter space.

We employed the 2D GMM clustering algorithm using two orbital parameters: the apogalactic distance $r_{\max }$ and the maximum height from the plane $|z|_{\max }$. For the GCs separation, we take into account the contribution of the set of orbits of each GC and the three bar pattern speeds. To prevent confusion in the clustering method, we removed the orbits with $r_{\max }>20 \mathrm{kpc}$.

From the GMM algorithm, we obtain the centre $\mu_{i}\left(r_{\max },|z|_{\max }\right)$, width $\sigma_{i}\left(r_{\max },|z|_{\max }\right)$, and weight $P_{i}$ of each stellar component. For the bulge/bar component $\mu_{b}=(2.50,1.07) \mathrm{kpc}, \sigma_{b}=(0.83$, $0.63) \mathrm{kpc}$, and $P_{b}=0.38$; for the thick disc $\mu_{d}=(5.60,2.42)$ $\mathrm{kpc}, \sigma_{d}=(1.29,1.09) \mathrm{kpc}$, and $P_{d}=0.46$; for the inner halo $\mu_{H_{i}}=(8.60,5.12) \mathrm{kpc}, \sigma_{H_{i}}=(1.31,1.02) \mathrm{kpc}$, and $P_{H_{i}}=0.11$, and outer halo $\mu_{H_{o}}=(12.75,8.47) \mathrm{kpc}, \sigma_{H_{o}}=(2.57,2.18) \mathrm{kpc}$, and $P_{H_{o}}=0.032$.

For this work, a statistical approach was necessary since the classification depends on the distribution of the data in the parameter space and their uncertainties. The GMM was applied from the library scikit-learn (Pedregosa et al. 2011). 
은ำ

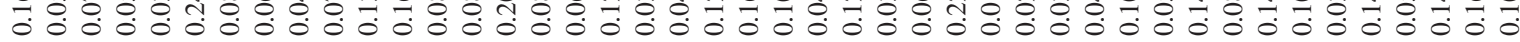
$H+H+H+H+H+H+H+H+H+H+H+H+H+H+H+H+H+H+H+H+H+H+H+H+H+H+H+H+H+H+H$

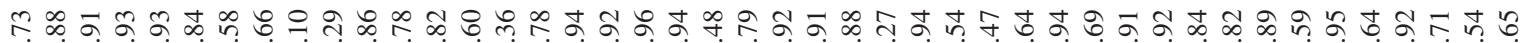

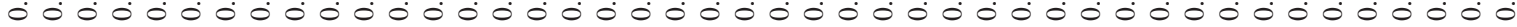

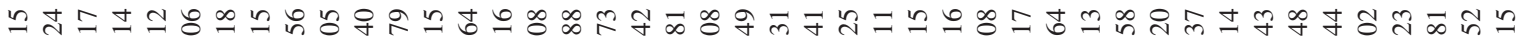

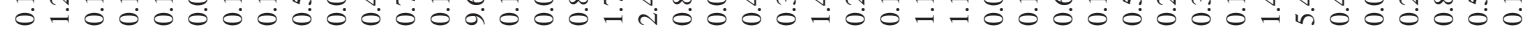
$H+H+H+H+H+H+H+H+H+H+H+H+H+H+H+H+H+H+H+H+H+H+H+H+H+H+H+H+H+H$

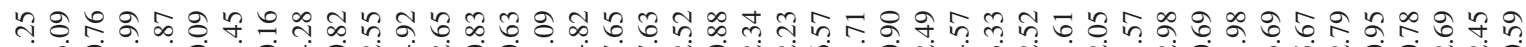

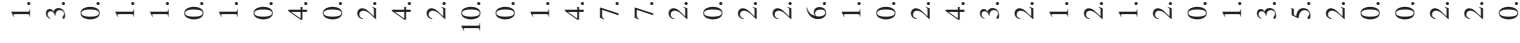

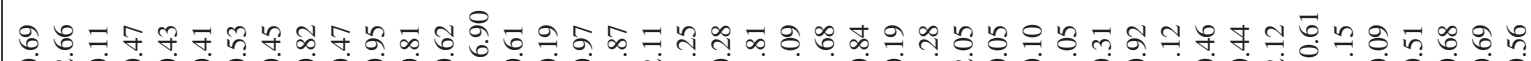

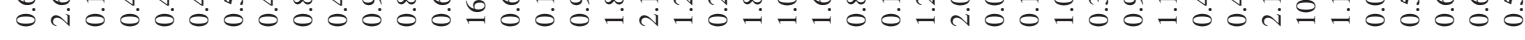
$H+H+H+H+H+H+H+H+H+H$ H H H H H H H H H H H H H H H H H H H H H H H H H H H H H H สำ ำ

드요의

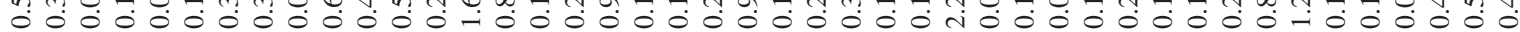

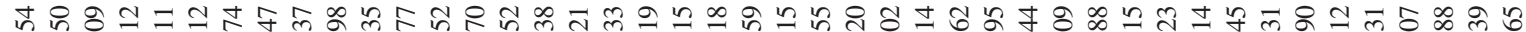

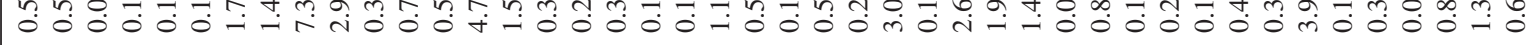

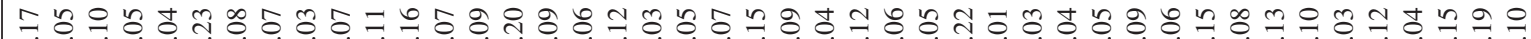

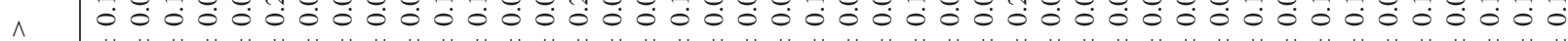
$\check{V} \quad H+H+H+H+H+H+H+H+H+H+H$ H H H H H H H H H H H H H H H H H H H H H H H H H H H H H 다유.

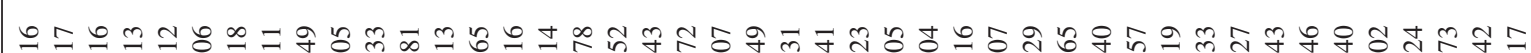

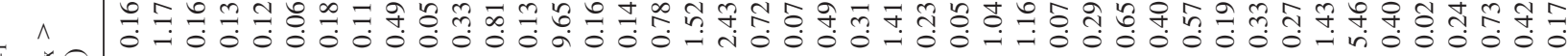

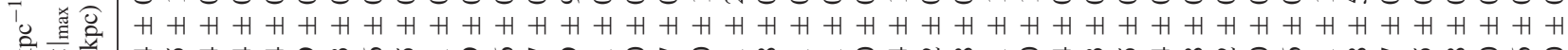

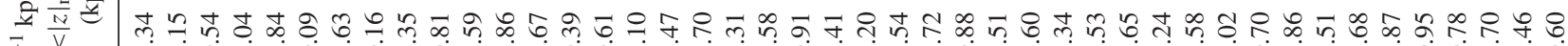
药 is 1

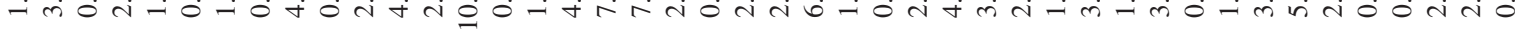

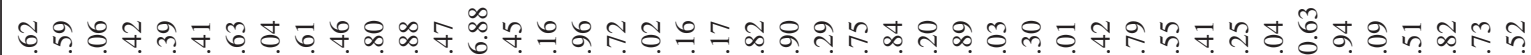
G

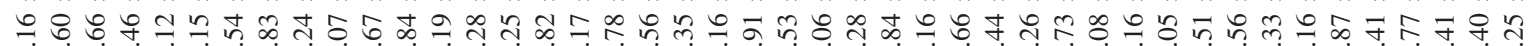
mím

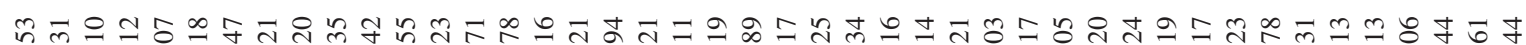

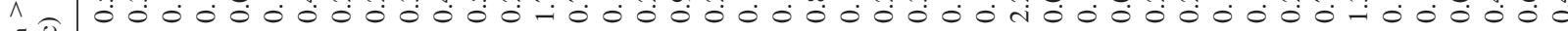
焉 H H H H H H H H H H H H H H H H H H H H H H H H H H H H H H H H H H H H H H H H H H H H $\stackrel{\mathrm{v}}{2}$

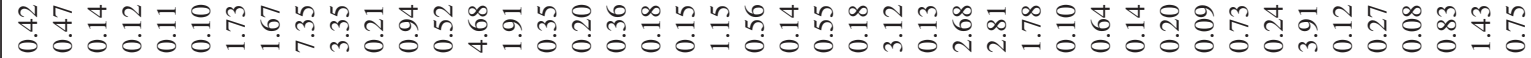

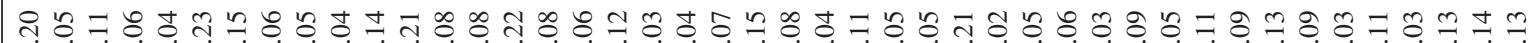
$\wedge$

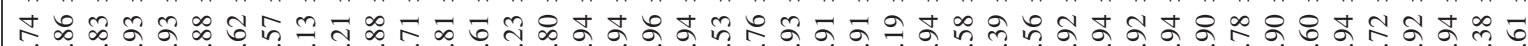

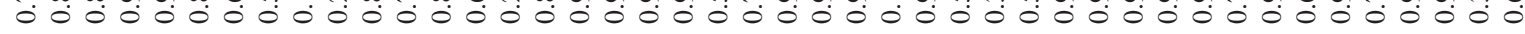

눙

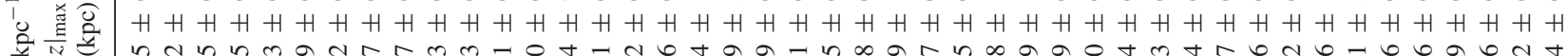
快 章 의의의 लि -

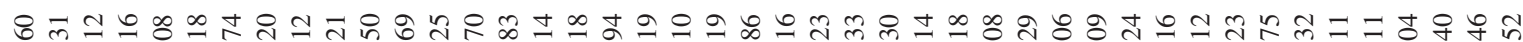
$\wedge$ o $000000000000.00000000000004000000000-000000$ H H H H H H H H H H H H H H H H H H H H H H H H H H H H H H H H H H H H H H H H H H H H 产

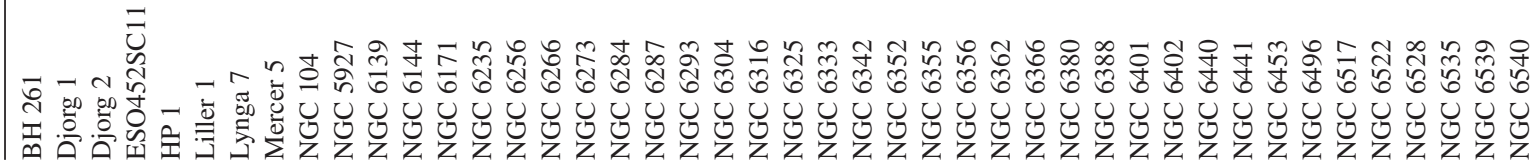




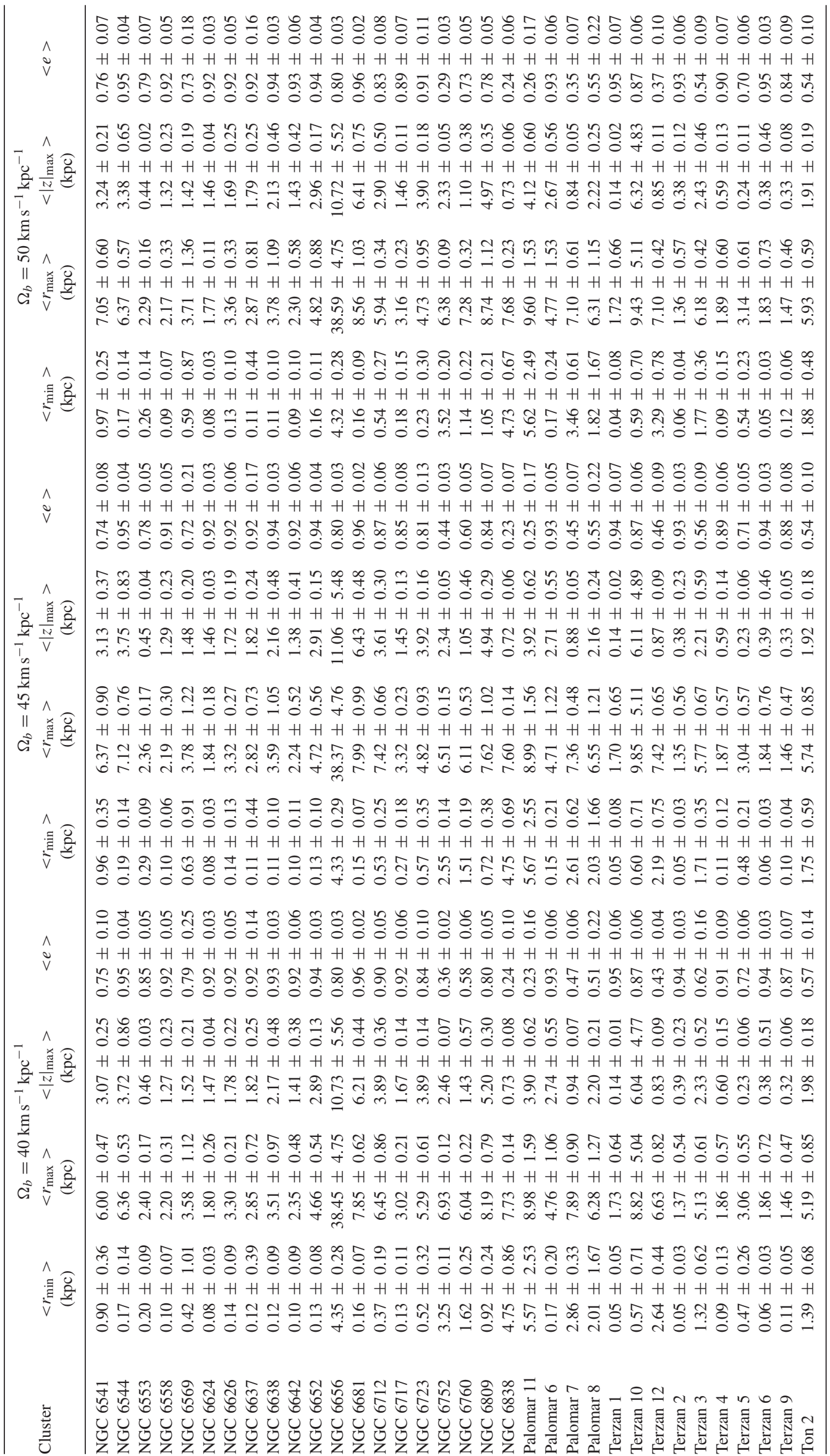



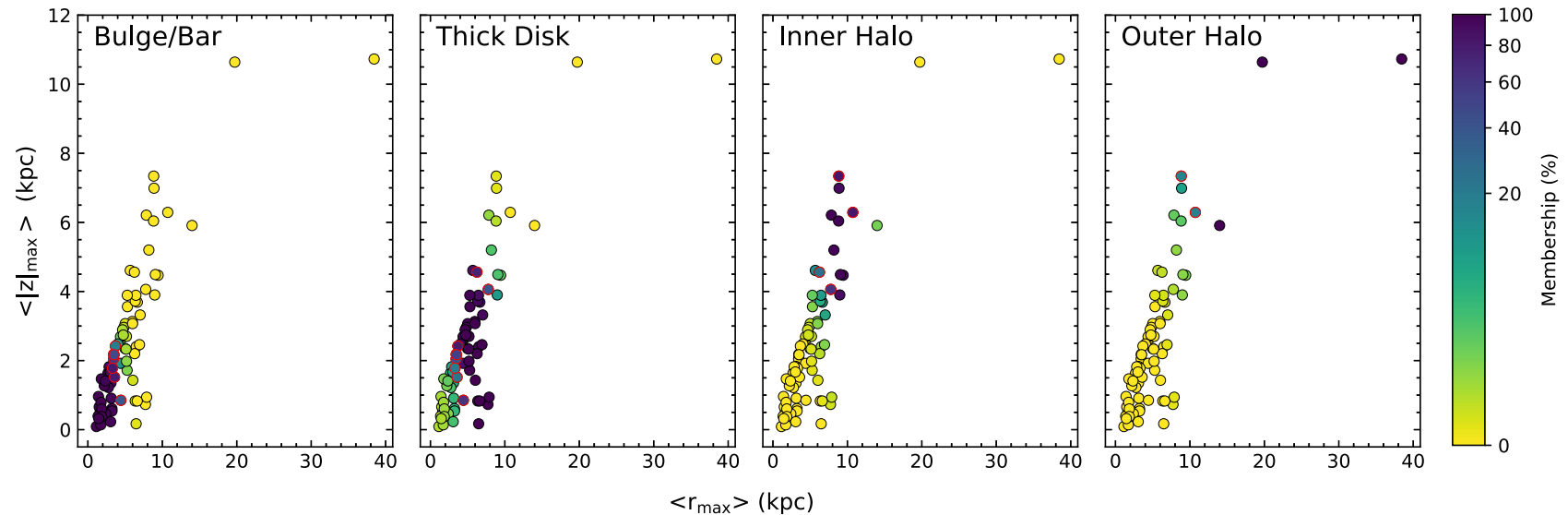

Figure 5. Membership probability for $\Omega_{b}=40 \mathrm{~km} \mathrm{~s}^{-1} \mathrm{kpc}^{-1}$. From left-hand to right-hand panel shows the probability that each GC has to belong to the bulge/bar, thick disc, inner halo, and outer halo, respectively. The open red circles highlight the clusters with probabilities between 15 per cent and 85 per cent that are in the boundary of two Galactic component.

Table 5. Membership probability for different bar pattern speed.

\begin{tabular}{|c|c|c|c|c|c|c|c|c|c|c|c|c|}
\hline \multirow{3}{*}{$\begin{array}{l}\text { Cluster } \\
\text { BH } 261\end{array}$} & \multicolumn{4}{|c|}{$\Omega_{b}=40 \mathrm{~km} \mathrm{~s}^{-1} \mathrm{kpc}^{-1}$} & \multicolumn{4}{|c|}{$\Omega_{b}=45 \mathrm{~km} \mathrm{~s}^{-1} \mathrm{kpc}^{-1}$} & \multicolumn{4}{|c|}{$\Omega_{b}=50 \mathrm{~km} \mathrm{~s}^{-1} \mathrm{kpc}^{-1}$} \\
\hline & \multirow{2}{*}{$\begin{array}{c}\text { Bulge } \\
94.2\end{array}$} & \multirow{2}{*}{$\begin{array}{r}\operatorname{Disc}_{\text {thick }} \\
\mathrm{p} \\
5.8\end{array}$} & \multirow{2}{*}{$\begin{array}{c}\text { Halo }_{\text {inner }} \\
\text { cent }\end{array}$} & \multirow{2}{*}{$\begin{array}{c}\text { Halo }_{\text {outer }} \\
0.0\end{array}$} & \multirow{2}{*}{$\begin{array}{l}\text { Bulge } \\
93.4\end{array}$} & \multicolumn{2}{|c|}{$\begin{array}{l}\text { Disc }_{\text {thick }} \text { Halo }_{\text {inner }} \\
\text { per cent }\end{array}$} & \multirow{2}{*}{$\begin{array}{c}\text { Halo outer }_{\text {on }} \\
0.0\end{array}$} & \multirow{2}{*}{$\begin{array}{l}\text { Bulge } \\
93.4\end{array}$} & \multicolumn{2}{|c|}{$\begin{array}{l}\text { Disc }_{\text {thick }} \text { Halo }_{\text {inner }} \\
\text { per cent }\end{array}$} & \multirow{2}{*}{$\begin{array}{r}\text { Halo }_{\text {oute }} \\
0.0\end{array}$} \\
\hline & & & & & & 6.6 & 0.0 & & & 6.6 & 0.0 & \\
\hline Djorg 1 & 0.0 & 93.4 & 6.6 & 0.0 & 0.0 & 88.7 & 11.3 & 0.0 & 0.0 & 93.2 & 6.8 & 0.0 \\
\hline Djorg 2 & 99.8 & 0.2 & 0.0 & 0.0 & 99.8 & 0.2 & 0.0 & 0.0 & 99.7 & 0.3 & 0.0 & 0.0 \\
\hline ESO452SC11 & 59.2 & 40.8 & 0.0 & 0.0 & 59.6 & 40.4 & 0.0 & 0.0 & 54.3 & 45.7 & 0.0 & 0.0 \\
\hline HP 1 & 86.5 & 13.5 & 0.0 & 0.0 & 85.4 & 14.6 & 0.0 & 0.0 & 87.6 & 12.4 & 0.0 & 0.0 \\
\hline Liller 1 & 99.8 & 0.2 & 0.0 & 0.0 & 99.8 & 0.2 & 0.0 & 0.0 & 99.8 & 0.2 & 0.0 & 0.0 \\
\hline Lynga 7 & 0.7 & 99.3 & 0.0 & 0.0 & 0.3 & 99.7 & 0.0 & 0.0 & 0.0 & 100.0 & 0.0 & 0.0 \\
\hline Mercer 5 & 0.0 & 100.0 & 0.0 & 0.0 & 0.0 & 100.0 & 0.0 & 0.0 & 0.0 & 100.0 & 0.0 & 0.0 \\
\hline NGC 104 & 0.0 & 1.2 & 98.0 & 0.8 & 0.0 & 2.3 & 97.1 & 0.6 & 0.0 & 3.5 & 96.0 & 0.5 \\
\hline NGC 5927 & 0.0 & 100.0 & 0.0 & 0.0 & 0.1 & 99.9 & 0.0 & 0.0 & 0.1 & 99.9 & 0.0 & 0.0 \\
\hline NGC 6139 & 0.8 & 99.2 & 0.0 & 0.0 & 0.5 & 99.4 & 0.0 & 0.0 & 0.8 & 99.1 & 0.0 & 0.0 \\
\hline NGC 6144 & 0.0 & 87.1 & 12.9 & 0.1 & 0.0 & 74.3 & 25.6 & 0.1 & 0.0 & 71.2 & 28.7 & 0.1 \\
\hline NGC 6171 & 0.0 & 99.9 & 0.1 & 0.0 & 0.1 & 99.9 & 0.1 & 0.0 & 0.1 & 99.9 & 0.0 & 0.0 \\
\hline NGC 6235 & 0.0 & 0.0 & 0.0 & 100.0 & 0.0 & 0.0 & 0.0 & 100.0 & 0.0 & 0.0 & 0.0 & 100.0 \\
\hline NGC 6256 & 96.0 & 4.0 & 0.0 & 0.0 & 95.9 & 4.1 & 0.0 & 0.0 & 94.5 & 5.5 & 0.0 & 0.0 \\
\hline NGC 6266 & 97.7 & 2.3 & 0.0 & 0.0 & 97.7 & 2.3 & 0.0 & 0.0 & 97.2 & 2.8 & 0.0 & 0.0 \\
\hline NGC 6273 & 0.0 & 73.9 & 26.1 & 0.1 & 0.0 & 80.3 & 19.6 & 0.1 & 0.0 & 55.5 & 44.3 & 0.1 \\
\hline NGC 6284 & 0.0 & 0.0 & 83.8 & 16.1 & 0.0 & 0.0 & 46.7 & 53.3 & 0.0 & 0.0 & 65.4 & 34.6 \\
\hline NGC 6287 & 0.0 & 0.0 & 91.7 & 8.3 & 0.0 & 0.0 & 74.7 & 25.3 & 0.0 & 0.0 & 58.8 & 41.2 \\
\hline NGC 6293 & 1.1 & 98.9 & 0.0 & 0.0 & 1.7 & 98.2 & 0.0 & 0.0 & 2.0 & 98.0 & 0.0 & 0.0 \\
\hline NGC 6304 & 96.3 & 3.7 & 0.0 & 0.0 & 96.0 & 4.0 & 0.0 & 0.0 & 94.7 & 5.3 & 0.0 & 0.0 \\
\hline NGC 6316 & 0.4 & 99.6 & 0.0 & 0.0 & 0.4 & 99.6 & 0.0 & 0.0 & 0.4 & 99.6 & 0.0 & 0.0 \\
\hline NGC 6325 & 49.3 & 50.7 & 0.0 & 0.0 & 43.3 & 56.7 & 0.0 & 0.0 & 44.7 & 55.3 & 0.0 & 0.0 \\
\hline NGC 6333 & 0.0 & 0.0 & 82.9 & 17.1 & 0.0 & 0.0 & 64.9 & 35.1 & 0.0 & 0.0 & 47.8 & 52.2 \\
\hline NGC 6342 & 86.6 & 13.4 & 0.0 & 0.0 & 83.7 & 16.3 & 0.0 & 0.0 & 82.7 & 17.3 & 0.0 & 0.0 \\
\hline NGC 6352 & 38.3 & 61.7 & 0.0 & 0.0 & 11.7 & 88.3 & 0.0 & 0.0 & 2.8 & 97.2 & 0.0 & 0.0 \\
\hline NGC 6355 & 6.7 & 93.3 & 0.0 & 0.0 & 4.1 & 95.9 & 0.0 & 0.0 & 2.6 & 97.4 & 0.0 & 0.0 \\
\hline NGC 6356 & 0.0 & 2.3 & 97.2 & 0.6 & 0.0 & 3.6 & 96.0 & 0.4 & 0.0 & 2.8 & 96.7 & 0.5 \\
\hline NGC 6362 & 0.0 & 91.9 & 8.1 & 0.0 & 0.0 & 99.5 & 0.5 & 0.0 & 0.0 & 99.6 & 0.4 & 0.0 \\
\hline NGC 6366 & 0.0 & 99.7 & 0.3 & 0.0 & 0.0 & 98.5 & 1.5 & 0.0 & 0.0 & 99.5 & 0.5 & 0.0 \\
\hline NGC 6380 & 95.8 & 4.2 & 0.0 & 0.0 & 95.4 & 4.6 & 0.0 & 0.0 & 94.1 & 5.9 & 0.0 & 0.0 \\
\hline NGC 6388 & 0.0 & 99.3 & 0.7 & 0.0 & 0.0 & 93.9 & 6.0 & 0.0 & 2.1 & 97.9 & 0.0 & 0.0 \\
\hline NGC 6401 & 90.3 & 9.7 & 0.0 & 0.0 & 90.1 & 9.9 & 0.0 & 0.0 & 90.5 & 9.5 & 0.0 & 0.0 \\
\hline NGC 6402 & 0.0 & 99.9 & 0.1 & 0.0 & 0.0 & 99.9 & 0.1 & 0.0 & 0.0 & 99.9 & 0.1 & 0.0 \\
\hline NGC 6440 & 99.8 & 0.2 & 0.0 & 0.0 & 99.8 & 0.2 & 0.0 & 0.0 & 99.8 & 0.2 & 0.0 & 0.0 \\
\hline NGC 6441 & 11.0 & 89.0 & 0.0 & 0.0 & 7.0 & 93.0 & 0.0 & 0.0 & 8.8 & 91.2 & 0.0 & 0.0 \\
\hline NGC 6453 & 0.0 & 99.4 & 0.6 & 0.0 & 0.0 & 99.4 & 0.6 & 0.0 & 0.0 & 95.2 & 4.8 & 0.0 \\
\hline NGC 6496 & 0.0 & 0.0 & 0.9 & 99.1 & 0.0 & 0.0 & 9.4 & 90.6 & 0.0 & 0.0 & 14.7 & 85.3 \\
\hline NGC 6517 & 0.1 & 99.9 & 0.1 & 0.0 & 0.1 & 99.9 & 0.1 & 0.0 & 0.1 & 99.8 & 0.0 & 0.0 \\
\hline NGC 6522 & 99.8 & 0.2 & 0.0 & 0.0 & 99.8 & 0.2 & 0.0 & 0.0 & 99.8 & 0.2 & 0.0 & 0.0 \\
\hline NGC 6528 & 99.7 & 0.3 & 0.0 & 0.0 & 99.7 & 0.3 & 0.0 & 0.0 & 99.7 & 0.3 & 0.0 & 0.0 \\
\hline NGC 6535 & 0.0 & 38.9 & 60.9 & 0.2 & 0.0 & 99.9 & 0.1 & 0.0 & 0.0 & 99.9 & 0.1 & 0.0 \\
\hline
\end{tabular}


Table 5 - continued

\begin{tabular}{|c|c|c|c|c|c|c|c|c|c|c|c|c|}
\hline \multirow{3}{*}{$\begin{array}{l}\text { Cluster } \\
\text { NGC } 6539\end{array}$} & \multicolumn{4}{|c|}{$\Omega_{b}=40 \mathrm{~km} \mathrm{~s}^{-1} \mathrm{kpc}^{-1}$} & \multicolumn{4}{|c|}{$\Omega_{b}=45 \mathrm{~km} \mathrm{~s}^{-1} \mathrm{kpc}^{-1}$} & \multicolumn{4}{|c|}{$\Omega_{b}=50 \mathrm{~km} \mathrm{~s}^{-1} \mathrm{kpc}^{-1}$} \\
\hline & \multirow{2}{*}{$\begin{array}{c}\text { Bulge } \\
19.5\end{array}$} & \multirow{2}{*}{$\begin{array}{r}\text { Disc }_{\text {thick }} \\
\mathrm{p} \\
80.5\end{array}$} & \multirow{2}{*}{ 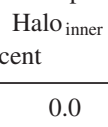 } & \multirow{2}{*}{$\begin{array}{c}\text { Halo outer } \\
0.0\end{array}$} & \multirow{2}{*}{$\begin{array}{r}\text { Bulge } \\
2.2\end{array}$} & \multicolumn{2}{|c|}{$\begin{array}{l}\text { Disc }_{\text {thick }} \mathrm{Halo}_{\text {inner }} \\
\text { per cent }\end{array}$} & \multirow{2}{*}{$\begin{array}{c}\text { Halo outer } \\
0.0\end{array}$} & \multirow{2}{*}{$\begin{array}{r}\text { Bulge } \\
0.5\end{array}$} & \multicolumn{2}{|c|}{$\begin{array}{c}\text { Disc }_{\text {thick }} \mathrm{Halo}_{\text {inner }} \\
\text { per cent }\end{array}$} & \multirow{2}{*}{$\begin{array}{r}\text { Halo }_{\text {outer }} \\
0.0\end{array}$} \\
\hline & & & & & & 97.8 & 0.0 & & & 99.5 & 0.0 & \\
\hline NGC 6541 & 0.0 & 99.4 & 0.6 & 0.0 & 0.0 & 98.6 & 1.4 & 0.0 & 0.0 & 94.2 & 5.7 & 0.0 \\
\hline NGC 6544 & 0.0 & 94.6 & 5.4 & 0.0 & 0.0 & 80.7 & 19.3 & 0.1 & 0.0 & 97.4 & 2.6 & 0.0 \\
\hline NGC 6553 & 99.3 & 0.7 & 0.0 & 0.0 & 99.4 & 0.6 & 0.0 & 0.0 & 99.4 & 0.6 & 0.0 & 0.0 \\
\hline NGC 6624 & 99.3 & 0.7 & 0.0 & 0.0 & 99.3 & 0.7 & 0.0 & 0.0 & 99.3 & 0.7 & 0.0 & 0.0 \\
\hline NGC 6626 & 81.1 & 18.9 & 0.0 & 0.0 & 82.4 & 17.6 & 0.0 & 0.0 & 81.8 & 18.2 & 0.0 & 0.0 \\
\hline NGC 6637 & 91.7 & 8.3 & 0.0 & 0.0 & 92.2 & 7.8 & 0.0 & 0.0 & 91.8 & 8.2 & 0.0 & 0.0 \\
\hline NGC 6638 & 46.7 & 53.3 & 0.0 & 0.0 & 42.3 & 57.7 & 0.0 & 0.0 & 32.1 & 67.9 & 0.0 & 0.0 \\
\hline NGC 6642 & 98.5 & 1.5 & 0.0 & 0.0 & 98.8 & 1.2 & 0.0 & 0.0 & 98.6 & 1.4 & 0.0 & 0.0 \\
\hline NGC 6652 & 0.2 & 99.8 & 0.0 & 0.0 & 0.1 & 99.8 & 0.0 & 0.0 & 0.1 & 99.9 & 0.1 & 0.0 \\
\hline NGC 6752 & 0.0 & 99.3 & 0.7 & 0.0 & 0.0 & 99.8 & 0.2 & 0.0 & 0.0 & 99.8 & 0.2 & 0.0 \\
\hline NGC 6760 & 0.0 & 100.0 & 0.0 & 0.0 & 0.0 & 99.9 & 0.0 & 0.0 & 0.0 & 100.0 & 0.0 & 0.0 \\
\hline NGC 6809 & 0.0 & 2.1 & 97.4 & 0.5 & 0.0 & 9.6 & 90.1 & 0.3 & 0.0 & 1.3 & 98.1 & 0.5 \\
\hline NGC 6838 & 0.0 & 100.0 & 0.0 & 0.0 & 0.0 & 100.0 & 0.0 & 0.0 & 0.0 & 100.0 & 0.0 & 0.0 \\
\hline Palomar 11 & 0.0 & 9.6 & 89.9 & 0.5 & 0.0 & 9.1 & 90.4 & 0.5 & 0.0 & 2.0 & 97.1 & 0.9 \\
\hline Palomar 6 & 0.2 & 99.8 & 0.0 & 0.0 & 0.3 & 99.7 & 0.0 & 0.0 & 0.3 & 99.7 & 0.0 & 0.0 \\
\hline Palomar 7 & 0.0 & 99.9 & 0.1 & 0.0 & 0.0 & 100.0 & 0.0 & 0.0 & 0.0 & 100.0 & 0.0 & 0.0 \\
\hline Palomar 8 & 0.0 & 99.9 & 0.1 & 0.0 & 0.0 & 99.8 & 0.2 & 0.0 & 0.0 & 99.9 & 0.1 & 0.0 \\
\hline Terzan 1 & 99.7 & 0.3 & 0.0 & 0.0 & 99.7 & 0.3 & 0.0 & 0.0 & 99.7 & 0.3 & 0.0 & 0.0 \\
\hline Terzan 10 & 0.0 & 0.1 & 98.2 & 1.7 & 0.0 & 0.0 & 95.3 & 4.7 & 0.0 & 0.0 & 95.9 & 4.1 \\
\hline Terzan 12 & 0.0 & 100.0 & 0.0 & 0.0 & 0.0 & 100.0 & 0.0 & 0.0 & 0.0 & 100.0 & 0.0 & 0.0 \\
\hline Terzan 2 & 99.8 & 0.2 & 0.0 & 0.0 & 99.8 & 0.2 & 0.0 & 0.0 & 99.8 & 0.2 & 0.0 & 0.0 \\
\hline Terzan 3 & 0.2 & 99.8 & 0.0 & 0.0 & 0.0 & 99.9 & 0.0 & 0.0 & 0.0 & 99.8 & 0.2 & 0.0 \\
\hline
\end{tabular}

\subsection{Membership probability and GC classification}

With the information of the each component provided by the GMM and using a Gaussian distribution probability, we calculate the membership probability that each cluster has to belong to each Galactic component. Fig. 5 shows the membership probability for $\Omega_{b}=40 \mathrm{~km} \mathrm{~s}^{-1} \mathrm{kpc}^{-1}$. The dark blue colour indicates the clusters that have probability higher than 85 percent to belong to the bulge/bar (first panel), thick disc (second panel), inner halo (third panel), and outer halo (four panel) component. The red open circles mark the clusters with probability between 15 per cent and 85 percent, that are in the boundary between two Galactic components. Table 5 gives the probability for each cluster to be part of the bulge/bar, disc, inner halo, and outer halo, for the three pattern speeds of the bar.

The maximum probability of membership given in Table 5 was used to classify the GCs into each Galactic component. Fig. 6 shows a plot of combinations among the orbital parameters, where the colours identify the groups of clusters based on the membership probability. Fig. 6 also shows a clear correlation between $<r_{\max }$ $>$ and $<|z|_{\max }>$, and an anticorrelation between $<r_{\min }>$ and eccentricity.

In Table 6, we list the GCs that belong to each of the Galactic components. From our sample has 40 of $43 \mathrm{GCs}$ identified as bulge
GCs in Bica et al. (2016), and based on their dynamical properties, for the pattern speed of $40 \mathrm{~km} \mathrm{~s}^{-1} \mathrm{kpc}^{-1}$, we found that 27 of them have characteristics of bulge GCs with a higher probability, whereas the other 13 GCs (Mercer 5, NGC 6316, NGC 6325, NGC 6355, NGC 6539, NGC 6638, NGC 6652, Ton 2, Palomar 6, Djorg 1, Terzan 3, Terzan 10, and NGC 6723) appear to be intruders from other stellar components, which currently are crossing the central parts of the Galaxy, provided that their distances are confirmed in the future. Additionally, the clusters NGC 6380 and NGC 6569 are part of the bulge GCs in our clasification, therefore we have 29 bulge GCs. Another 37, 9, and 3 GCs are identified as thick disc, inner halo, and outer halo, respectively. There are three clusters, NGC 6535, NGC 6284, and NGC 6333, that change component membership with the bar pattern speed. Additionally, there are clusters with significant probability to be in two stellar components. ESO452SC11, NGC 6325, NGC 6352, NGC 6539, NGC 6569, NGC 6626, and NGC 6638 are in the boundary between bulge/bar and thick disc; NGC 6273 and NGC 6535 are between thick disc and inner halo; NGC 6284 and NGC 6333 are between inner halo and outer halo, and these clusters could change with the bar pattern speed. It is important to stress that due to the uncertainties of the data, in particular in the distances, the classification established here, might change in the future when better distance determinations are available. One example is Palomar 6, that with the distance of $\mathrm{d}_{\odot}$ 

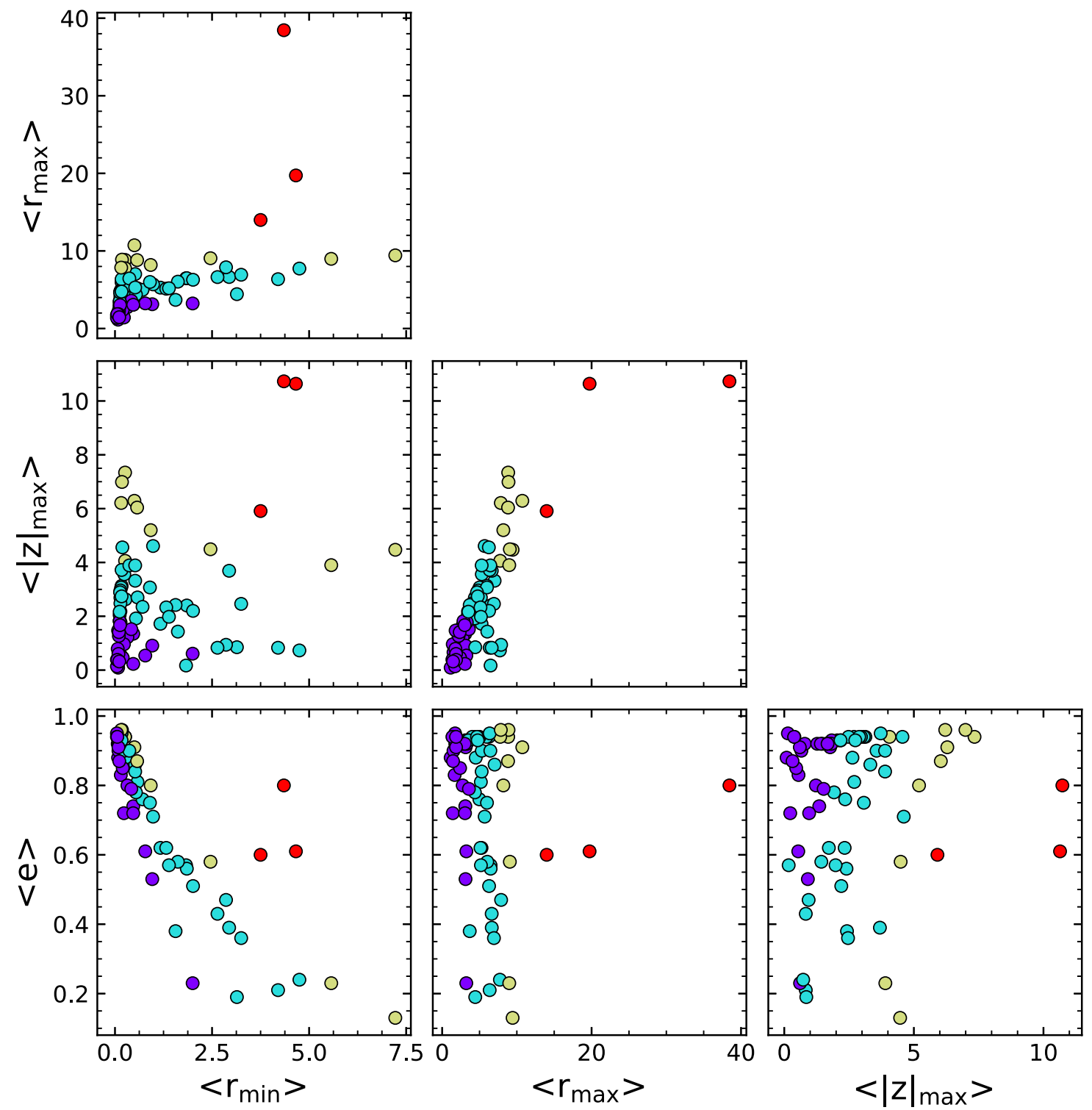

Figure 6. Orbital parameters as function of the median values of the perigalactic distance $<r_{\min }>$, the apogalctic ditance $\left\langle r_{\max }>\right.$, the maximum distance from the Galactic plane $<|z|_{\max }>$, and eccentricity $<e>$, for $\Omega_{b}=40 \mathrm{~km} \mathrm{~s}^{-1} \mathrm{kpc}^{-1}$. The four groups are bulge/bar (purple), thick disc (cyan), inner halo (green), and outer halo (red).

$=5.8 \mathrm{kpc}$ puts it in the thick disc component, whereas the distance by Ortolani, Bica $\&$ Barbuy (1995) of $\mathrm{d}_{\odot}=8.9 \mathrm{kpc}$ puts it in the bulge.

Fig. 7 shows the spatial distribution of the sample GCs, based on our classification and the stellar component with higher membership probability, where we can note that GCs from different Galactic components overlap in the innermost part of the Galaxy.

\section{BULGE GLOBULAR CLUSTERS}

Once, we identify clusters with high probability to be bulge GCs, we analyse their set of orbits, with the purpose of knowing which bulge GCs follow the bar. To classify orbits, we use the same criteria defined by Portail, Wegg \& Gerhard (2015b). The orbit classification employs frequency analysis. First, we compute the fast Fourier transform for each orbit in the Cartesian $x$-coordinate and the Cylindrical radius $R$, to identify the main frequencies. Then, the orbits for which the frequency ratio $f_{R} / f_{x}=2 \pm 0.1$ are barfollowing orbits. The orbits that are not supporting the bar shape have a frequency ratio $f_{R} / f_{x} \neq 2 \pm 0.1$. Table 7 gives the percentage of orbits in each bulge GC that support the bar shape. The clusters Liller 1, NGC 6304, NGC 6522, NGC 6528, NGC 6540, NGC 6553, Terzan 5, and Terzan 9 have more than 20 per cent of their orbits that support the bar. The fraction of orbits that follow the Galactic bar decreases with the rotation of the bar, except for the cases of NGC 6304, NGC 6342, and NGC 6637, which increases instead. Also, we found that most of the bulge/bar GCs are not suporting the Galactic bar.

On the other hand, there are bulge GCs that even if they are not supporting the bar shape, they are trapped into a bar 
Table 6. Classification of GCs based on dynamical properties, assuming their maximum membership probability.

\begin{tabular}{|c|c|c|c|}
\hline \multirow{2}{*}{$\frac{\text { Component }}{\text { Bulge/Bar }}$} & \multicolumn{3}{|c|}{ Clusters } \\
\hline & BH 261 & NGC 6401 & NGC 6637 \\
\hline & Djorg 2 & NGC 6440 & NGC 6642 \\
\hline & ESO452SC11 & NGC 6522 & NGC 6717 \\
\hline & HP 1 & NGC 6528 & Terzan 1 \\
\hline & Liller 1 & NGC 6540 & Terzan 2 \\
\hline & NGC 6256 & NGC 6553 & Terzan 4 \\
\hline & NGC 6266 & NGC 6558 & Terzan 5 \\
\hline & NGC 6304 & NGC 6569 & Terzan 6 \\
\hline & NGC 6342 & NGC 6624 & Terzan 9 \\
\hline & NGC 6380 & NGC 6626 & \\
\hline \multirow[t]{13}{*}{ Thick disc } & Djorg 1 & NGC 6388 & NGC 6712 \\
\hline & Lynga 7 & NGC 6362 & NGC 6723 \\
\hline & Mercer 5 & NGC 6366 & NGC 6752 \\
\hline & NGC 5927 & NGC 6402 & NGC 6760 \\
\hline & NGC 6139 & NGC 6441 & NGC 6838 \\
\hline & NGC 6144 & NGC 6453 & Palomar 6 \\
\hline & NGC 6171 & NGC 6517 & Palomar 7 \\
\hline & NGC 6273 & NGC $6535^{*}$ & Palomar 8 \\
\hline & NGC 6293 & NGC 6539 & Terzan 12 \\
\hline & NGC 6316 & NGC 6541 & Terzan 3 \\
\hline & NGC 6325 & NGC 6544 & Ton 2 \\
\hline & NGC 6352 & NGC 6638 & \\
\hline & NGC 6355 & NGC 6652 & \\
\hline \multirow[t]{3}{*}{ Inner halo } & NGC 104 & NGC 6356 & Palomar 11 \\
\hline & NGC 6284* & NGC 6681 & Terzan 10 \\
\hline & NGC 6287 & NGC 6809 & NGC 6333* \\
\hline Outer halo & NGC 6235 & NGC 6496 & NGC 6656 \\
\hline
\end{tabular}

${ }^{*}$ GCs that its classification changes with $\Omega_{b}$.

resonance such as NGC 6266 and NGC 6558, in the 3:1 resonance.

Additionally, the metallicity distribution function of the classified bulge GCs is shown in Fig. 8, where we can note a clear peak at $[\mathrm{Fe} / \mathrm{H}] \sim-1.0$, as previously already reported by Rossi et al.

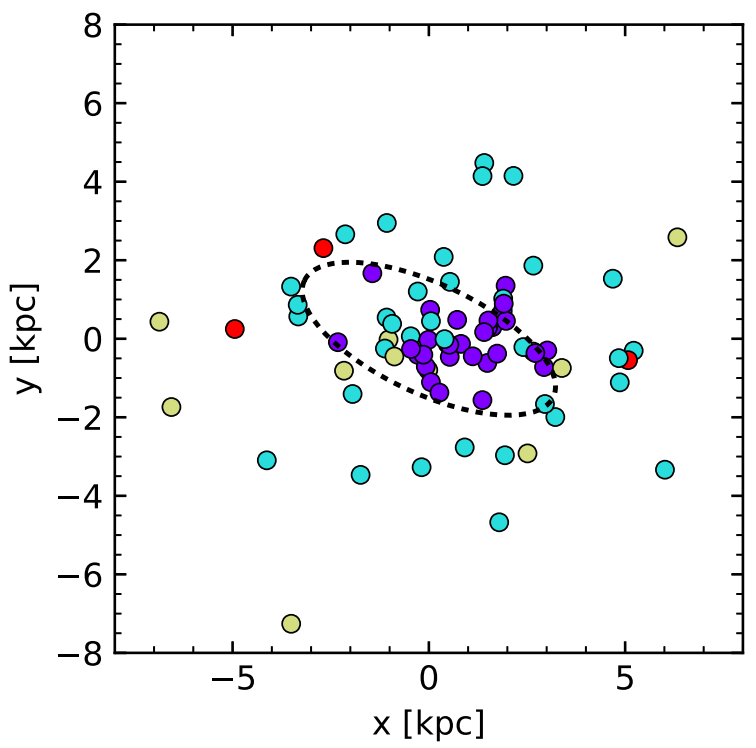

Table 7. Bulge GC orbits that follow the Galactic bar for different pattern speed.

\begin{tabular}{|c|c|c|c|}
\hline \multirow[t]{2}{*}{ Cluster } & \multicolumn{3}{|c|}{$\begin{array}{l}\text { Orbits following the bar } \\
\text { (per cent) }\end{array}$} \\
\hline & $\Omega_{b}=40$ & $\Omega_{b}=45$ & $\Omega_{b}=50$ \\
\hline BH 261 & 6.2 & 6.9 & 8.9 \\
\hline Djorg 2 & 0.0 & 0.1 & 0.0 \\
\hline ESO452SC 11 & 0.0 & 0.2 & 0.0 \\
\hline HP 1 & 0.2 & 0.4 & 0.8 \\
\hline Liller 1 & 38.2 & 32.1 & 28.1 \\
\hline NGC 6256 & 9.0 & 19.7 & 36.1 \\
\hline NGC 6266 & 13.1 & 9.9 & 8.3 \\
\hline NGC 6304 & 27.8 & 38.1 & 38.4 \\
\hline NGC 6342 & 1.0 & 3.1 & 3.5 \\
\hline NGC 6380 & 0.7 & 0.9 & 0.3 \\
\hline NGC 6401 & 0.7 & 0.7 & 1.4 \\
\hline NGC 6440 & 11.3 & 9.1 & 7.5 \\
\hline NGC 6522 & 97.0 & 95.3 & 94.7 \\
\hline NGC 6528 & 44.0 & 24.0 & 7.8 \\
\hline NGC 6540 & 23.1 & 18.2 & 12.9 \\
\hline NGC 6553 & 65.2 & 63.3 & 57.0 \\
\hline NGC 6558 & 19.3 & 12.5 & 6.9 \\
\hline NGC 6569 & 2.0 & 2.6 & 3.1 \\
\hline NGC 6624 & 0.3 & 0.0 & 0.1 \\
\hline NGC 6626 & 0.4 & 0.1 & 0.5 \\
\hline NGC 6637 & 1.3 & 4.2 & 4.4 \\
\hline NGC 6642 & 7.2 & 2.8 & 2.1 \\
\hline NGC 6717 & 0.7 & 0.8 & 0.7 \\
\hline Terzan 1 & 2.0 & 2.3 & 1.6 \\
\hline Terzan 2 & 0.0 & 0.0 & 0.0 \\
\hline Terzan 4 & 19.6 & 15.1 & 13.7 \\
\hline Terzan 5 & 32.8 & 25.0 & 12.3 \\
\hline Terzan 6 & 1.7 & 2.1 & 0.5 \\
\hline Terzan 9 & 87.7 & 81.8 & 73.1 \\
\hline
\end{tabular}

(2015b) and Bica et al. (2016). Fig. 9 gives the metallicity as a function of the median value of the orbital parameters, the perigalactic and apogalactic distances, the maximum height, and the eccentricity. We cannot see any trend among orbital parameters

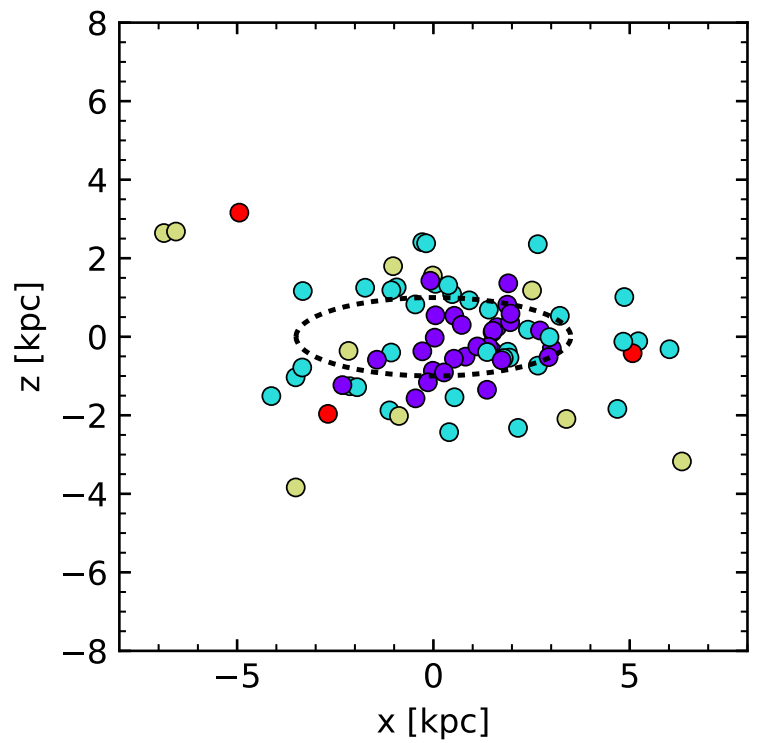

Figure 7. Spatial distribution of the GCs in the $x-y$ (left-hand panel) and $x-z$ (right-hand planel) projection. The colours are the same as in Fig. 6 and indicate the stellar component to which GCs are associated. The black dashed line shows the size of the Galactic bar. 


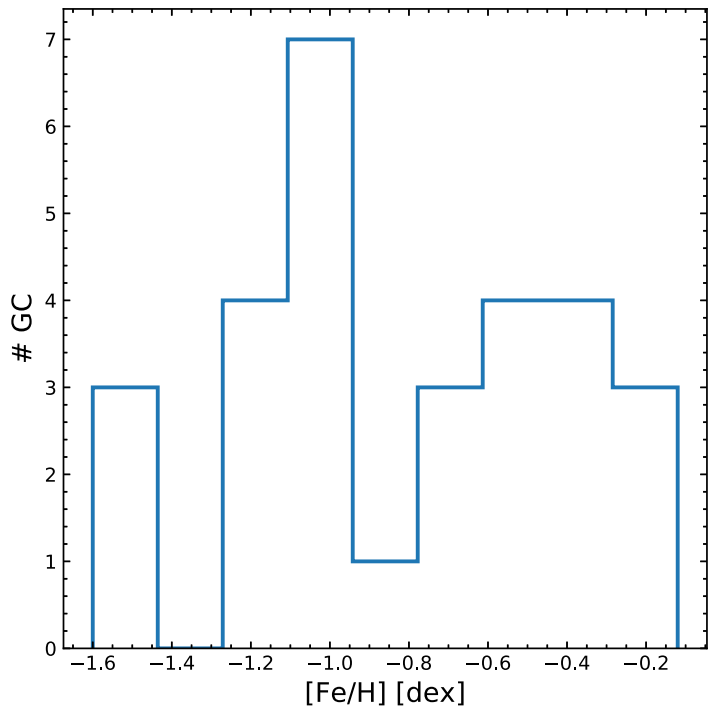

Figure 8. Metallicity distribution of bulge globular clusters.

with the metallicity. Also, the effect of changing the bar pattern speed is almost negligible for the bulge GCs.

\section{CONCLUSIONS}

Combining accurate absolute PMs from Gaia DR2 with distances from RR Lyrae and/or CMD when available, and radial velocities from spectroscopy, we were able to construct the orbits of $78 \mathrm{GCs}$ among the most centrally located in the Galaxy, using a Galactic bar potential. Most sample clusters are located in the innermost region of the MW.

We generate a set of initial conditions for each cluster using Monte Carlo simulations, taking into account the uncertainties from the heliocentric distance and kinematic data of the clusters. We calculated the median value of the orbital parameters such as the perigalactic and apogalactic distances, the maximum vertical excursion from the Galactic plane, and the eccentricity. Through the dynamical properties of the orbits, apogalactic distance, and the maximum height from the plane, we applied a clustering technique to estimate the membership probability that each cluster has to be part of different stellar populations: bulge/bar, thick disc, inner halo, or outer halo.
Given that in the inner part of the MW, most of Galactic components overlap, one of the goals of this study was to identify which among the bulge GCs classified as such by Bica et al. (2016), are confined in the bulge/bar region. In a few cases, like Djorg 1 and Terzan 10, they are identified as halo intruders in the Galactic bulge (Ortolani et al. 2019a), and with our analysis, we confirm that they are in fact contaminating the bulge GC population. Others in the same case are NGC 6316, NGC 6355, Ton 2, Palomar 6, and NGC 6723, provided that their distances are confirmed. In terms of dynamical properties, the orbital characteristics of the GCs identified to be part of the bulge/bar are distinct from those in the thick disc/inner halo according to the present classification criteria.

The GCs are very old, whereas the bar likely formed later. In the already seminal paper by Sheth et al. (2008), it was shown that whereas 65 per cent of the present-day luminous spiral galaxies have bars, at redshift $z \sim 0.84$ (about $6 \mathrm{Gyr}$ ago), this fraction drops to 20 per cent at redshift $z=0$. From a fully cosmological simulation, Buck et al. (2018) suggest that the Galaxy's bar formed $8 \pm 2 \mathrm{Gyr}$ ago. Similarly, Bovy et al. (2019) used chemical abundances from the Apache Point Observatory Galactic Evolution Experiment survey, and kinematical information from the Gaia collaboration, and concluded that the Galactic bar formed $\sim 8 \mathrm{Gyr}$ ago. Therefore, the fact that GCs are in the bar, probably indicates that they were confined in the bar when the latter formed.

Most of the bulge GCs are confined in the bar region but are not supporting the bar structure, and the seven clusters that are supporting the bar do not necessarily support the X-shape. In particular, Terzan 5, which is confined in the bar, was revealed to have multiple populations in a range of metallicities and ages (Origlia et al. 2019, and references therein), suggesting this object to be the nucleus of a dwarf galaxy.

Currently, we have very accurate proper motions from Gaia and radial velocities from high-resolution spectroscopy, however, in most cases there are no accurate distance determinations, and they are crucial to characterize the orbits. Further efforts on distance derivation from accurate CMDs and/or RR Lyrae, are greatly needed.

\section{ACKNOWLEDGEMENTS}

We acknowledge the anonymous referee for the detailed review and for the helpful suggestions, which allowed us to improve the manuscript. APV acknowledges the FAPESP postdoctoral
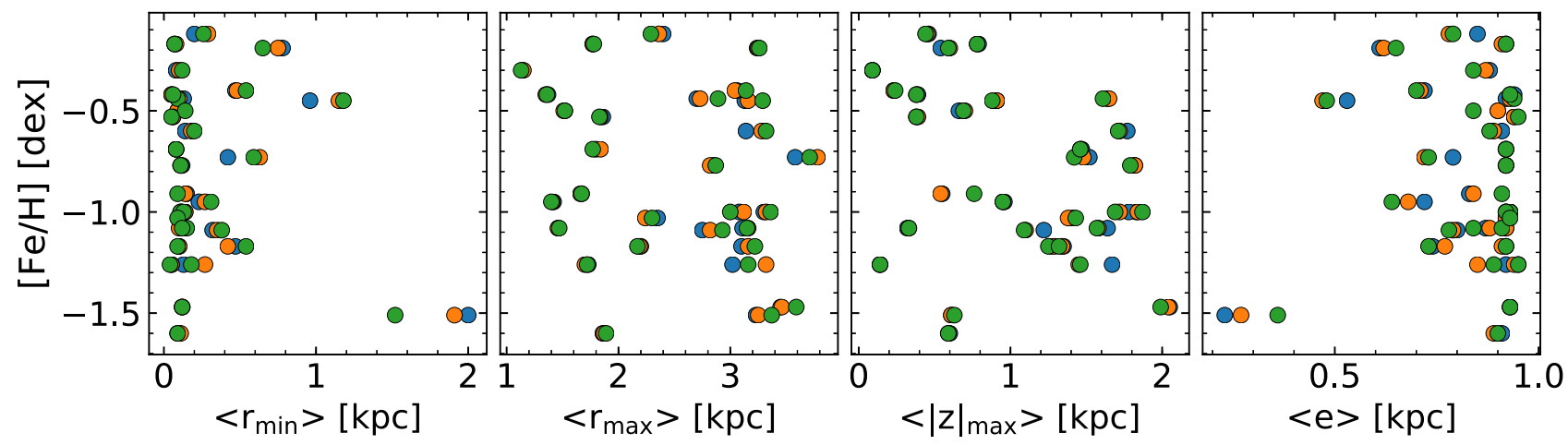

Figure 9. Metallicity of bulge globular clusters as a function of the median values of perigalactic distance $<r_{\min }>$ (first panel), apogalctic distance $<r_{\max }>$ (second panel), maximum distance from the Galactic plane $<|z|_{\max }>$ (third panel), and eccentricity $<e>$ (fourth panel), for $\Omega_{b}=40$ (blue), 45 (orange), and 50 (green) $\mathrm{km} \mathrm{s}^{-1} \mathrm{kpc}^{-1}$. 
fellowship no. 2017/15893-1. BB and EB acknowledge grants from the Brazilian agencies CAPES - Finance code 001, CNPq, and FAPESP. SO acknowledges partial support by the Università degli Studi di Padova Progetto di Ateneo CPDA141214 and BIRD178590 and by INAF under the program PRIN-INAF2014. SOS acknowledges the FAPESP PhD fellowship no. 2018/22044-3. APV and SOS acknowledge the DGAPA-PAPIIT grant IG100319. 'This work has used data from the European Space Agency (ESA) mission Gaia (https://www.cosmos.esa.int/gaia), processed by the Gaia Data Processing and Analysis Consortium (DPAC, https: //www.cosmos.esa.int/web/gaia/dpac/consortium). Funding for the DPAC has been provided by national institutions, in particular the institutions participating in the Gaia Multilateral Agreement.'

\section{REFERENCES}

Aguilar L., Hut P., Ostriker J. P., 1988, ApJ, 335, 720

Armandroff T. E., 1989, AJ, 97, 375

Barbuy B., Bica E., Ortolani S., 1998, A\&A, 333, 117

Barbuy B., et al., 2014, A\&A, 570, A76

Barbuy B., et al., 2016, A\&A, 591, A53

Barbuy B., Chiappini C., Gerhard O., 2018a, ARA\&A, 56, 223

Barbuy B., et al., 2018b, A\&A, 619, A178

Baumgardt H., Hilker M., Sollima A., Bellini A., 2019, MNRAS, 482, 5138

Bica E., Bonatto C., Barbuy B., Ortolani S., 2006, A\&A, 450, 105

Bica E., Ortolani S., Barbuy B., 2016, Publ. Astron. Soc. Aust., 33, e028

Bica E., Pavani D. B., Bonatto C. J., Lima E. F., 2019, AJ, 157, 12

Bissantz N., Englmaier P., Gerhard O., 2003, MNRAS, 340, 949

Bland-Hawthorn J., Gerhard O., 2016, ARA\&A, 54, 529

Bovy J., Leung H. W., Hunt J. A. S., Mackereth J. T., García-Hernández D. A., Roman-Lopes A., 2019, MNRAS, 490, 740

Buck T., Ness M. K., Obreja A., Maccio A. V., Dutton A. A., 2018, ApJ, 861,88

Caldwell N., Romanowsky A. J., 2016, ApJ, 824, 42

Côté P., 1999, AJ, 118, 406

Dias B., Barbuy B., Saviane I., Held E. V., Da Costa G. S., Ortolani S., Gullieuszik M., Vásquez S., 2016, A\&A, 590, A9

Dinescu D. I., Girard T. M., van Altena W. F., López C. E., 2003, AJ, 125, 1373

Frenk C., White S., 1982, MNRAS, 198, 173

Freudenreich H. T., 1998, ApJ, 492, 495

Gaia Collaboration, 2018a, A\&A, 616, A1

Gaia Collaboration, 2018b, A\&A, 616, A12

Gardner E., Flynn C., 2010, MNRAS, 405, 545

Harris W. E., 1996, AJ, 112, 1487

Hilker M., Baumgardt H., Sollima A., Bellini A., 2019, preprint (arXiv: 1908.02778)
Irrgang A., Wilcox B., Tucker E., Schiefelbein L., 2013, A\&A, 549, A137

Kent S. M., Dame T. M., Fazio G., 1991, ApJ, 378, 131

Kerber L. O., Nardiello D., Ortolani S., Barbuy B., Bica E., Cassisi S., Libralato M., Vieira R. G., 2018, ApJ, 853, 15

Kerber L. O. et al., 2019, MNRAS, 484, 5530

Minniti D., 1995, AJ, 109, 1663

Minniti D., Alonso-García J., Pullen J., 2017a, Res. Notes Am. Astron. Soc., 1,54

Minniti D. et al., 2017b, ApJ, 849, L24

Miyamoto M., Nagai R., 1975, PASJ, 27, 533

Navarro J. F., Frenk C. S., White S. D. M., 1997, ApJ, 490, 493

Origlia L. et al., 2019, ApJ, 871, 114

Ortolani S., Bica E., Barbuy B., 1995, A\&A, 296, 680

Ortolani S., Nardiello D., Pérez-Villegas A., Bica E., Barbuy B., 2019a, A\&A, 622, A94

Ortolani S. et al., 2019b, A\&A, 627, A145

Pasquato M., Chung C., 2019, MNRAS, 490, 3392

Pedregosa F., Varoquaux G., Gramfort A. et al., 2011, JMLR, 12, 2825

Pérez-Villegas A., Portail M., Wegg C., Gerhard O., 2017, ApJ, 840, L2

Pérez-Villegas A., Rossi L., Ortolani S., Casotto S., Barbuy B., Bica E., 2018, Publ. Astron. Soc. Aust., 35, e021

Pfenniger D., 1984, A\&A, 134, 373

Portail M., Wegg C., Gerhard O., Martinez-Valpuesta I., 2015a, MNRAS, 448, 713

Portail M., Wegg C., Gerhard O., 2015b, MNRAS, 450, L66

Portail M., Gerhard O., Wegg C., Ness M., 2017, MNRAS, 465, 1621

Rattenbury N. J., Mao S., Sumi T., Smith M. C., 2007, MNRAS, 378, 1064

Rossi L. J., 2015a, Astron. Comput., 12, 11

Rossi L. J., Ortolani S., Barbuy B., Bica E., Bonfanti A., 2015b, MNRAS, 450,3270

Saito R. K. et al., 2012, A\&A, 537, A107

Schönrich R., Binney J., Dehnen W., 2010, MNRAS, 403, 1829

Sheth K. et al., 2008, ApJ, 675, 1141

Smith R., Flynn C., Candlish G. N., Fellhauer M., Gibson B. K., 2015, MNRAS, 448, 2934

Valenti E., Ferraro F. R., Origlia L., 2007, AJ, 133, 1287

Valenti E., Ferraro F. R., Origlia L., 2010, MNRAS, 402, 1729

Vasiliev E., 2019, MNRAS, 484, 2832

Vásquez S. et al., 2018, A\&A, 619, A13

Wegg C., Gerhard O., 2013, MNRAS, 435, 1874

Weiner B. J., Sellwood J. A., 1999, ApJ, 524, 112

Zinn R., 1980, ApJ, 241, 602

Zinn R., 1985, ApJ, 293, 424

This paper has been typeset from a $\mathrm{T}_{\mathrm{E}} \mathrm{X} / \mathrm{L} \mathrm{T} \mathrm{T} \mathrm{X}$ file prepared by the author. 